Relations industrielles

Industrial Relations

\title{
L'amnistie des fautes disciplinaires
}

\section{Claude D'Aoust}

Volume 43, numéro 4, 1988

URI : https://id.erudit.org/iderudit/050457ar

DOI : https://doi.org/10.7202/050457ar

Aller au sommaire du numéro

Éditeur(s)

Département des relations industrielles de l'Université Laval

ISSN

0034-379X (imprimé)

1703-8138 (numérique)

Découvrir la revue

Citer cet article

D'Aoust, C. (1988). L'amnistie des fautes disciplinaires. Relations industrielles / Industrial Relations, 43(4), 909-942. https://doi.org/10.7202/050457ar
Résumé de l'article

L'auteur étudie l'effet atténuant de l'écoulement du temps qui, à la limite, rend inopposable aujourd'hui une faute qu'on aurait du reprocher hier au salarie qui lui mérite une sanction.
Tous droits réservés ㄷ Département des relations industrielles de l'Université Laval, 1988
Ce document est protégé par la loi sur le droit d'auteur. L’utilisation des services d'Érudit (y compris la reproduction) est assujettie à sa politique d'utilisation que vous pouvez consulter en ligne.

https://apropos.erudit.org/fr/usagers/politique-dutilisation/ 


\section{DROIT DU TRAVAIL}

\section{L'amnistie des fautes disciplinaires}

\section{Claude D'Aoust}

L'auteur étudie l'effet atténuant de l'écoulement du temps qui, à la limite, rend inopposable aujourd'hui une faute qu'on aurait dû reprocher hier au salarié qui lui mérite une sanction.

L'amnistie des fautes du salarié en efface les conséquences, en ce qu'elles sont restées impunies, ou que la sanction, partiellement ou entièrement purgée, n'est plus retenue contre lui'. L'un et l'autre type ont un point commun: dans un cas comme dans l'autre la faute est pardonnée, oubliée, de sorte que l'employeur ${ }^{2} n^{\prime}$ est pas autorisé par la suite à reprocher son manquement au salarié comme motif unique ou concurrent de sanction ${ }^{3}$.

Il s'agit donc de la renonciation au droit de sanctionner l'inexécution d'une obligation ou, dans le second cas, de l'abandon du droit d'invoquer un manquement déjà sanctionné comme facteur de détermination de la sanction appropriée lors d'un manquement subséquent, qu'il soit ou non de même nature.

Le mot «sanction» est employé dans son sens le plus large, ${ }^{4}$ comprenant aussi bien la notion de droit civil que celle du droit pénal's. Si l'on songe à l'autorité patronale en tant que pouvoir de répression, il s'agira de punition devant être imposée, en regard du principe de progressivité des sanctions, dès que la faute est connue de l'employeur, de manière que le salarié sache que son action est réprouvée et que la récidive entraînera des sanctions plus lourdes encore; conséquemment, en vertu du même principe, la première sanction pourra être invoquée plus tard sans violation du principe non bis in idem (prohibition de la double sanction).

* D'AOUST, C. École de relations industrielles, Université de Montréal.

** L'auteur remercie sa collaboratrice, madame Inès Mairet, qui s'est chargée de la recherche de la jurisprudence. Ce travail a été réalisé à l'aide d'une subvention du Conseil de recherches en sciences humaines du Canada. 
Même dans sa dimension civile, le manquement (considéré comme inexécution de l'obligation de fournir une prestation convenable) doit être signalé au salarié, sans quoi ce dernier serait autorisé à tenir pour acquis, après un certain temps et surtout si son insuffisance est continue, que son obligation a été allégée par accord tacite, interprétation qui s'accorderait avec la théorie de la révision du contrat de travail ${ }^{6}$.

On pourrait aussi arguer que si l'employeur est habilité à se faire justice à lui-même en renvoyant le salarié jugé inapte à fournir une prestation normale, le caractère exceptionnel de cette faculté de résiliation unilatérale exigerait qu'elle soit exercée avec discernement et donc que le salarié soit, en un premier temps, mis en demeure i.e. prévenu de l'insatisfaction de l'employeur en regard de son rendement et des conséquences de la persistance du manquement ${ }^{7}$. Il est vrai qu'en droit strict un débiteur en défaut peut être poursuivi en justice sans avertissement; ${ }^{8}$ encore faut-il considérer que dans le cas qui nous occupe l'employeur est à la fois juge et partie. L'équité commanderait, en raison de ce fait, un adoucissement des règles du droit commun des obligations.

Au moment donc de prendre une sanction, l'employeur peut et doit considérer le passé et non pas seulement la faute contemporaine, immédiate, commise par le salarié. Si ce dernier a un «dossier chargé», ce facteur jouera contre lui. Par contre, l'attitude passée de l'employeur, qui n'aurait pas réagi à ses manquements au bon moment, jouera en faveur du salarié. Sauf quand la faute mérite le renvoi immédiat en raison de son extrême gravité, la détermination de la sanction appropriée requiert la constitution d'une «histoire de cas». Dans cet article, nous étudierons l'effet atténuant de l'écoulement du temps qui, à la limite, rend inopposable aujourd'hui une faute qu'on aurait dû reprocher hier au salarié ou - en certains cas - qui lui a même mérité une sanction.

\section{L'AMNISTIE DE LA FAUTE AVANT SANCTION}

L'amnistie avant sanction est expresse ou implicite.

\section{L'amnistie expresse}

Mise à part l'amnistie législative dont nous avons déjà parlé, (voir la note 2), de même que le règlement des griefs par le protocole de retour au travail, ou par transaction, l'amnistie expresse avant sanction ne pose aucun problème, au plan théorique. Elle n'est assujettie à aucune formalité, pas 
plus que le processus disciplinaire lui-même 9 . Elle peut être orale ou écrite, mais l'écrit est préférable, du point de vue du salarié, car la preuve en sera plus facile en cas de litige.

Si l'amnistie avant sanction intervient dans le cadre d'une procédure interne de règlement des griefs, ${ }^{10}$ les formalités de cette dernière devront être respectées; toutefois, cette procédure ne traite le plus souvent que du cheminement de la discussion défini en termes de temps (délais) et de niveau hiérarchique tant de l'entreprise que du syndicat (étapes), gardant le silence sur l'acte de règlement lui-même ${ }^{11}$.

S'inscrit également dans la catégorie de l'amnistie explicite l'adoption de mesures patronales ne constituant pas des mesures disciplinaires. L'employeur qui, connaissant le manquement, se contente de signaler la faute au salarié sans la sanctionner, ne peut par la suite revenir sur sa position; il n'en est pas empêché par la règle non bis in idem (ou prohibition de la double sanction), puisque par hypothèse aucune sanction n'a été imposée au départ, mais plutôt par le fait qu'il a d'abord suivi une ligne de conduite incompatible avec l'expression ultérieure d'une attitude répressive. En d'autres termes, l'employeur ne peut absoudre le salarié de sa faute tout en la retenant contre lui.

Certes, aussi longtemps que le délai pour sévir n'est pas expiré, l'employeur pourra changer d'avis dans la mesure où une action première n'impliquait pas nécessairement le pardon du manquement ${ }^{12}$. Ainsi, une observation à la suite d'une faute peut, selon les circonstances, s'interpréter comme un constat qui, après étude, conduira à une sanction ou plutôt apparaîtra comme une mesure définitive comportant renonciation à l'exercice du pouvoir disciplinaire. S'il y a grief, l'arbitre appréciera les circonstances et en déduira la nature de la décision.

Mais la nature ou la portée de la mesure prise peut n'être mise en question que plus tard, à l'occasion d'un grief de congédiement par exemple. Une remarque verbale remontant à plusieurs mois - et par hypothèse autrement admissible en preuve - est-elle ou non une mesure disciplinaire ${ }^{13}$ ?

Supposons qu'une observation verbale ait été faite à un certain moment et que le dossier du salarié en comporte la relation écrite. Supposons aussi que la convention collective comprenne une liste exhaustive de sanctions ${ }^{14}$. Supposons enfin que cette liste ne mentionne ni avis verbal, ni réprimande, ni observation. Le syndicat pourrait alors s'opposer à ce que la preuve de l'incident soit faite en vue d'une preuve d'antécédents défavorables (i.e. à l'effet que le principe de progressivité a été respecté et qu'un incident culminant justifiait le renvoi), s'il s'agit d'une sanction pour motifs 
(purement) disciplinaires. Par contre, la preuve d'absences répétées, mais demeurées impunies, serait admissible (et la preuve en serait facilitée par la relation écrite que l'employeur en aurait faite au dossier) lorsqu'un salarié est renvoyé en raison d'une incapacité professionnelle causée par la maladie. Ces notes ne feraient donc pas partie du dossier disciplinaire du salarié, mais le contenu en serait admissible en preuve ${ }^{15}$. Bien plus, les informations relatives aux absences englobées dans les données générales du service de la rémunération, pourront être individualisées au besoin, ce qui montre qu'à l'ère informatique le dossier disciplinaire en tant qu'ensemble de données relatives à un salarié est une notion complexe. En d'autres termes, les données individuelles se retrouvent sous différentes formes et en divers services de l'entreprise. L'information conservée à une fin (par exemple, les données relatives à la rémunération destinées à la vérification comptable) peut au besoin être utilisée à une autre fin (par exemple, la rémunération versée à un individu sur une période servant à calculer son assiduité sur la même période) ${ }^{16}$.

Si les critères distinctifs de la mesure disciplinaire se trouvent dans la convention collective, ils sont déterminants. Autrement, il faut s'en remettre à ceux définis en jurisprudence arbitrale. Ainsi, la sanction disciplinaire se reconnaît à ce qu'elle est notée au dossier en vue d'une utilisation éventuelle au détriment du salarié ${ }^{17}$. L'intention de l'employeur est révélée non seulement par la teneur de la mesure, mais aussi par des éléments objectifs comme l'inclusion au dossier. Il apparaît donc que ces critères permettront également de décider si une observation écrite correspond à l'avis écrit que peut prévoir la convention collective i.e. si la mesure adoptée correspond à la définition qu'en donne implicitement ou explicitement la convention collective ${ }^{18}$.

Il ressort de la jurisprudence que la mesure disciplinaire se reconnaît au but visé, soit la correction du manquement reproché au salarié ${ }^{19}$. Un simple constat des faits, fût-il consigné par écrit au dossier, n'est pas une mesure disciplinaire ${ }^{20}$. Diverses actions peuvent être considérées comme disciplinaires, et la nature d'une mesure dépend des circonstances ${ }^{21}$. L'arbitre Fernand Morin l'avait déjà noté; il ajoute que «deux éléments caractéristiques [sont] fondamentaux: sa cause et sa finalité»» ${ }^{22}$. Il écrit:

[...] il doit y avoir une faute, un manquement, un écart de conduite professionnelle de la part du salarié, et d'autre part, la mesure doit servir, sans égard à son effet réel, à réprouver cette faute et à en faire cesser la répétition par l'auteur initial ou par les autres salariés $[\ldots] .^{23}$

Cette définition implicite de la mesure disciplinaire nous convient toutà-fait, précisément par son caractère abstrait qui lui confère la généralité essentielle à une définition. À partir de là, indiquer au salarié la méthode 
corrective désirée ou l'avertir des conséquences d'une récidive apparaissent comme des éléments circonstanciels dont la présence témoigne de l'existence de la cause et de la finalité mentionnés plus haut; en même temps, leur absence ne serait pas déterminante, car d'autres faits ou circonstances pourraient servir à établir l'existence de cette cause et de cette finalité.

Pour ce qui est de sa teneur, la sanction disciplinaire contient un élément de blâme, de désapprobation, de censure, de critique et (le plus souvent, mais est-ce un élément essentiel?) une indication des corrections désirées et des conséquences qu'entraînerait la persistance dans la commission de la faute reprochée.

La jurisprudence arbitrale n'est pas uniforme en la matière et tous les arbitres ne reconnaissent pas le caractère essentiel de tous les éléments déjà mentionnés ou, peut-être serait-ce plus exact, ne leur accordent pas la même importance relative ${ }^{24}$. De plus, on ne saurait se fier à une seule caractéristique de la mesure examinée; ainsi, l'avertissement verbal peut avoir un sens variant en fonction d'un certain nombre de facteurs. À cet égard, l'arbitre R.D. Joyce propose une intéressante typologie des avis oraux: ${ }^{25}$

«1) L'avis verbal de type 1 informe le salarié des attentes de l'employeur; il équivaut à la publication du règlement d'entreprise. La preuve en est permise mais elle établit uniquement que le salarié connaissait la règle.

2) L'avis verbal de type 2 est informel et n'est pas noté au dossier. Il vise à signaler un manquement à une règle déjà connue et à corriger la situation. Il ne donne pas ouverture à un grief non plus qu'à une preuve de progressivité des fautes et des sanctions.

3) L'avis verbal de type 3 est noté au dossier et fait partie de la discipline progressive. Par conséquent, il donne ouverture à la procédure de grief. ${ }^{26}$

Naturellement, il ne s'agit pas d'une typologie correspondant à des variétés d'avis prévues dans les conventions collectives. S'il y a une clause à cet effet, elle a préséance sur toute autre définition ou catégorie analytique. La typologie proposée par Joyce a son utilité pour analyser les mesures prises par l'employeur dans une situation donnée et ensuite en tirer les conséquences juridiques, e.g. déterminer si l'action de l'employeur a valeur d'amnistie. En effet, toute communication orale au salarié ne constitue pas nécessairement un avis disciplinaire et la communication orale non disciplinaire équivaut, s'il y a eu manquement disciplinaire, à une amnistie.

\section{L'amnistie implicite}

L'amnistie implicite se déduit quant à elle de la conduite de l'employeur. La forme la plus simple est celle qui découle de l'inaction combinée à l'écoulement du temps, une sorte de prescription extinctive du «droit» ${ }^{27}$ d'imposer une sanction à la suite d'une conduite jugée fautive. 
Cette «prescription» peut découler de la loi. C'est ainsi que le premier alinéa de l'article L. 122-44 du Code du travail français édicte:

Aucun fait fautif ne peut donner lieu à lui seul à l'engagement de poursuites disciplinaires au-delà d'un délai de deux mois à compter du jour où l'employeur en a eu connaissance, à moins que ce fait ait donné lieu dans le même délai à l'exercice de poursuites pénales. $[\ldots]$

Malgré une rédaction insolite (e.g. "poursuites disciplinaires»), cet article signifie que l'employeur doit exercer son pouvoir disciplinaire dans les deux mois du jour où il a pris connaissance du fait fautif.

Au Québec, le Code du travail n'a pas de disposition semblable. Il faut pourtant mentionner l'article 71 qui se lit:

Les droits et recours qui naissent d'une convention collective ou d'une sentence qui en tient lieu se prescrivent par six mois à compter du jour où la cause de l'action a pris naissance. Le recours à la procédure de griefs interrompt la prescription.

L'arrêt Sucre Atlantic applique cette règle supplétive à toute phase de la procédure d'arbitrage ${ }^{28}$. Mais peut-on donner à la règle une portée si générale qu'elle s'applique également aux droits (par opposition aux recours ou droits d'action, selon la distinction classique)? Et si tel était le cas, pourrait-on dire aussi que le pouvoir disciplinaire est un «droit né de la convention collective ${ }^{29}$ ? Une réponse affirmative à ces deux questions conduirait à conclure que la faute impunie dans les six mois de la connaissance qu'en a l'employeur ne pourrait plus l'être; il y aurait prescription. À l'inverse, l'employeur pourrait agir à la limite du délai et aucun plaidoyer de négligence ne serait recevable à l'encontre de ce comportement, que ce soit en vue de substituer un délai raisonnable au délai légal, ou pour obtenir l'annulation de la décision prise en violation des garanties de procédure protégeant le salarié.

Nous ne soutenons pas que l'article 71 C.t. a une portée aussi large que celle que nous venons d'évoquer. Cette hypothèse est soulevée pour étude et réflexion. Si toutefois cette source formelle de droit est écartée, il reste que des conventions collectives ont établi une prescription. Le Décret tenant lieu de convention collective en vertu de la Loi 105. SPPSCQ-SPPASQ-CSN ( 2 avril $1982-31$ décembre 1985) en comporte un exemple:

12.03 La décision d'imposer un congédiement ou une suspension est communiquée dans les trente (30) jours de l'incident y donnant lieu ou au plus tard dans les trente (30) jours de la connaissance par l'employeur de tous les faits pertinents liés à cet incident.

Le délai de trente (30) jours prévu à l'alinéa précédent ne s'applique pas si la décision d'imposer un congédiement ou une suspension résulte de la répétition de certains faits ou d'un comportement chronique du salarié. 
D'autres clauses sont encore plus explicites et précisent l'effet de l'expiration du délai. Par exemple:

Dans tous les cas de mesure disciplinaire, l'Employeur doit informer le salarié par écrit de la mesure disciplinaire qui lui est imposée en mentionnant l'incident qui motive la mesure disciplinaire. Copie d'un tel avis sera également donnée à l'Union et/ou au représentant de l'Union dans les meilleurs délais.

Pour être valide et être opposable au salarié, l'employeur doit mettre à la poste, pour le bénéfice de l'Union, dans les dix (10) jours ouvrables qui suivent les faits ayant donné ouverture à ladite mesure disciplinaire, une copie de ladite mesure disciplinaire prise contre le salarié. ${ }^{30}$

Passé le délai, l'employeur ne peut plus sévir. Il ne peut non plus faire la preuve de fautes ainsi impunies, lors de l'arbitrage relatif à des fautes postérieures.

En présence d'une règle écrite, il faut s'en remettre aux termes de la convention collective; seulement en cas d'obscurité ou d'ambiguité de ceuxci peut-on recourir aux règles d'interprétation des contrats ou des lois. La seule véritable question est ici de savoir si les délais prévus, par le simple écoulement du temps, créent des droits ou éteignent des obligations, touchant par là le fond même du litige ${ }^{31}$.

L'obligation de l'employeur de sévir sans délai dès qu'il a connaissance du manquement du salarié est bien établie en jurisprudence arbitrale ${ }^{32}$.

L'employeur, certes, peut invoquer divers motifs expliquant son retard à agir, mais tous ne l'excusent pas. Ainsi, le désir de sauvegarder la paix industrielle ne justifie pas un délai de onze semaines à sanctionner la participation à une grève illégale, ${ }^{33}$ non plus que l'examen des faits reprochés devant une instance judiciaire ${ }^{34}$.

Lorsque l'inaction de l'employeur a pour but de ne pas compromettre l'enquête mise sur pied pour découvrir la source de vols de marchandises, un arbitre a jugé que le retard à sévir est permis à condition que le salarié $n^{\prime}$ 'en souffre pas ${ }^{35}$. Cela revient à affirmer que le droit du salarié à une «défense pleine et entière» (qui fonde son droit d'être informé sans délai des reproches formulés contre lui et éventuellement de contester la sanction dans un délai raisonnable suivant la survenance des faits) prime la protection du droit de propriété de l'employeur i.e. une garantie procédurale (associée à une liberté fondamentale) a ici priorité sur un droit de nature civile, droit de propriété ou droit de créance, ce qui est dans l'ordre naturel des choses. Cependant, un arbitre américain a déjà décidé, avec raison selon nous, que la décision de l'employeur dans une telle situation constitue un choix entre deux lignes de conduite s'excluant mutuellement ${ }^{36}$. Si l'enquête porte sur un seul salarié, l'employeur ne pourrait non plus observer et noter faute sur faute sans réagir, dans le but d'accumuler des éléments de preuve 
pour justifier le congédiement $;{ }^{37} \mathrm{si}$ la première faute relevée ne justifie pas le congédiement et qu'une fois prévenu, voire puni, le salarié ne récidive pas, tout est bien qui finit bien car, en ce cas, la discipline corrective aura atteint son but.

Enfin, l'employeur ne peut invoquer l'ignorance d'un fait fautif connu de l'enquêteur désigné par lui ${ }^{38}$. Que l'on analyse la question dans le cadre du mandat ${ }^{39}$ ou de la délégation de pouvoir, ${ }^{40}$ le principe demeure que l'employeur, qui déjà soupçonne un manquement puisqu'il mène une enquête, doit procéder avec diligence de manière à ne pas porter préjudice à un salarié éventuellement puni. Il doit donc choisir avec soin les personnes chargées de l'enquête et leur donner instruction d'agir elles-mêmes diligemment. Si elles ne remplissent pas correctement cette obligation contractuelle, le principe de la relativité des contrats ${ }^{41}$ empêche que des tiers, les salariés, en subissent les conséquences.

L'employeur, donc, doit réagir à un manquement dès qu'il en a connaissance ou qu'il aurait pu ou dû en prendre connaissance.

\section{Amnistie et manquements non disciplinaires}

Le manquement non disciplinaire («innocent», «non-culpable», «non blameworthy conduct») pose un problème particulier. S'il ne paraît pas discutable que l'employeur doit lui notifier son insuffisance professionnelle avant de pouvoir renvoyer le salarié, le contenu et la nature de l'avis requis méritent d'être précisés. Par cette démarche, l'employeur informe le salarié de l'existence du problème et de la correction à y apporter et le prévient des conséquences qu'aurait la continuation de l'état de choses constaté ${ }^{42}$. Cette double exigence s'explique par un impératif de fair-play (il convient de mettre le salarié en garde avant qu'il ne perde irrémédiablement toute possibilité de rétablir la situation) ${ }^{43}$. D'autres soutiennent que l'avertissement est nécessaire à la preuve que l'insuffisance reprochée au salarié est irrémédiable ${ }^{44}$. Que l'on retienne cette explication ou non, il reste que l'irréversibilité de l'incapacité doit être prouvée; la seule controverse sur ce point concerne l'attribution du fardeau de la preuve ${ }^{45}$. Une troisième explication veut que l'avis informe le salarié des normes de rendement (standard of performance), celles-ci n'étant pas nécessairement connues, contrairement à la règle abstraite, qui est très claire; la difficulté réside justement dans la transposition du comportement du bon père de famille à une situation concrète et, pour la pallier, l'employeur doit informer le salariéét6.

On s'est aussi demandé si l'employeur pouvait imposer des mesures disciplinaires en guise d'avertissement et d'étapes préalables au renvoi pour 
motif non disciplinaire. Les arbitres sont partagés sur la question. Certains opinent que l'employeur ne peut d'aucune manière utiliser son pouvoir disciplinaire ${ }^{47}$. Par contre, d'autres arbitres ont reconnu à l'employeur le droit de sévir à l'occasion d'une faute non imputable au salarié ${ }^{48}$. De plus, un arbitre a reconnu que le fait qu'un avis mentionne l'éventualité de mesures disciplinaires à défaut d'une meilleure assiduité ne lui conférait pas un caractère disciplinaire; un tel avis est donc valable, même si les absences fréquentes s'expliquent par la maladie ${ }^{49}$.

Dans ces conditions, ${ }^{50}$ quelle conduite doit tenir l'employeur qui, sans pour autant chercher à se départir des services d'un salarié, veut se ménager une défense convenable au cas où il devrait en arriver à cette ultime mesure? Il paraît raisonnable de conclure qu'il peut procéder à l'avertissement et, plus tard, au congédiement, si la situation ne s'améliore pas: cette voie est celle qui se rapproche le plus du régime de droit commun dans lequel l'employeur se fait justice à lui-même ${ }^{51}$. La seule condition qu'impose la jurisprudence arbitrale est que le salarié soit d'abord informé de l'insatisfaction de l'employeur, sans égard à la forme que prend cet avertissement.

Par contre, si l'employeur juge plus opportune la voie disciplinaire, il graduera son intervention en fonction de l'évolution du problème, selon les règles connues de la progressivité des sanctions disciplinaires, échappant ainsi à l'alternative inexorable de l'autre procédé qui veut que le problème trouve une solution complète ou que le salarié soit renvoyé à brève échéance. Ainsi le salarié será averti, muté, peut-être rétrogradé, voire même suspendu, avant d'être renvoyée ${ }^{52}$. Cette façon de faire met l'accent sur la correction du problème plutôt que sur l'élimination de sa cause par le congédiement; entretemps, le salarié peut contester chacune de ces mesures, tout en conservant son poste.

\section{L'opposabilité de la faute amnistiée}

La question se pose de savoir si un manquement qui n'a pas été sanctionné - soit que l'employeur n'y ait pas donné suite ou qu'il ait pris une mesure inappropriée - peut être reproché au salarié à l'occasion d'un manquement subséquent. Plusieurs décisions arbitrales y ont répondu par l'affirmative $^{53}$. L'explication est que l'amnistie serait sujette à la condition implicite d'un comportement ultérieur satisfaisant ${ }^{54}$.

Notons cependant que le principe n'est pas fermement reconnu par la common law: on remarque un certain flottement dans la jurisprudence et des hésitations doctrinales ${ }^{55}$. En jurisprudence arbitrale, il semblerait plutôt que l'employeur n'est pas en principe recevable à prouver les manquements 
antérieurs non sanctionnés pour démontrer juste cause ${ }^{56}$. Il n'y aurait exception à cette règle que si le salarié présentait une preuve d'états de services antérieurs, affirmant ou laissant entendre que son dossier antérieur est impeccable ${ }^{57}$. L'exception se fonderait sur une renonciation implicite du salarié au bénéfice de la clause d'amnistie ou encore sur le droit qu'a une partie, ici l'employeur, de pouvoir contester tout fait allégué ou tout moyen de droit soulevé par son adversaire.

Ces remarques relatives à l'admissibilité en preuve du dossier antérieur (au sens large) paraissent également valables, sans égard au caractère disciplinaire ou non disciplinaire des manquements reprochés au salarié. Mais il faut ajouter quelques précisions pour ces derniers cas.

D'abord, si le salarié occupe ses fonctions depuis longtemps - des mois, voire des années - et que soudainement on le congédie ou on le mute en invoquant son insuffisance professionnelle, les difficultés de preuve seront accrues d'autant ${ }^{58}$. Le fait que le salarié ait occupé son poste durant une longue période sans être inquiété crée une présomption à l'effet qu'il a accompli ses fonctions de manière satisfaisante; à moins que l'incapacité ne résulte d'une cause subite et récente, cette présomption peut s'avérer difficile à renverser. Cette présomption n'est que relative; mais, certains arbitres considèrent que l'employeur ne peut congédier brusquement le salarié incapable. L'avertissement préalable apparaît alors comme une formalité essentielle dont le non-respect entraînerait la nullité de la mesure prise. Il faut donc bien distinguer ici l'effet des garanties procédurales, jugé absolu par certains arbitres, et l'effet de la présomption de capacité qui découle des faits et ne peut donc qu'être relative. Par ailleurs, d'aucuns affirment que l'employeur doit aussi avoir essayé de reclasser le salarié dans une catégorie d'emploi qui est dans sa sphère de compétence ${ }^{59}$. Cette opinion n'est pas fondée en droit commun, ni en droit disciplinaire. Du point de vue du droit des contrats, la partie incapable sans sa faute de fournir la prestation convenue n'est tenue à aucun dommage mais l'autre partie est elle-même libérée de ses obligations, en tous cas si le contrat est synallagmatique, et le contrat individuel de travail possède indubitablement ce caractère. Néanmoins, la règle de droit a été assouplie considérablement dans la pratique et il y a de nombreux exemples où la jurisprudence s'est montrée disposée à soulager les employeurs des contraintes apparues après la passation du contrat; ${ }^{60}$ on voit mal pourquoi l'assouplissement des règles ne jouerait pas également en faveur des salariés. Les décisions relatives au congédiement déguisé en sont un exemple; le maintien du lien contractuel en faveur du salarié devenu incapable de faire le travail pour lequel il a été embauché en serait un autre. Si le droit positif évoluait dans cette direction, le salarié conserverait son emploi dans la mesure où il serait apte à faire un travail et à condition qu'un poste correspondant soit disponible ${ }^{61}$. 
L'employeur peut congédier un salarié pour cause juste et suffisante et l'incapacité professionnelle est reconnue comme telle. Certes, l'employeur est tenu à certaines garanties procédurales dont il a été fait état plus haut. Les tempéraments à la règle de l'exécution de la prestation convenue concernent non pas la modification de la nature de cette prestation mais les circonstances de l'espèce ${ }^{62}$. Obliger l'employeur à offrir un poste adapté aux capacités diminuées du salarié est peut-être souhaitable, mais cette exigence ne découle pas du droit positif tel qu'il existe présentement.

La preuve du dossier antérieur n'est pertinente que dans le cadre d'un incident culminant. Celui-ci a deux caractéristiques fondamentales: non seulement c'est un manquement récent (ou en tous cas le plus récent des manquements imputés au salarié), mais encore est-ce un incident qui, ajouté à d'autres qui le précèdent, forme un tout équivalant à une cause juste et suffisante. C'est dire que si le dernier manquement forme à lui seul un motif juste de congédiement, le recours à la doctrine de l'incident culminant n'est pas indispensable, sinon inutile ${ }^{63}$.

D'autre part, le manquement continu est, en matière d'amnistie, à distinguer de celui qui se concrétise en événements distincts, donc identifiables dans le temps. L'amnistie ne vaut que pour le passé. Par conséquent, le manquement continu peut être sanctionné aussi longtemps qu'il dure (et donc à tout moment, s'il est permanent), à condition de le notifier au salarié et de lui accorder un délai raisonnable pour rectifier la situation. Encore faut-il observer que cette analyse procède de l'esprit du droit disciplinaire et que l'analyse de droit contractuel conduit à des conclusions différentes, voire opposées, par application de la théorie de la révision du contrat de travail ${ }^{64}$.

\section{L'AMNISTIE APRÈS SANCTION}

Une fois la faute dûment sanctionnée, ${ }^{65}$ il n'est pas dit qu'elle doive demeurer indéfiniment au passif du salarié. Le temps qui guérit les blessures atténue pareillement les effets d'une faute inscrite à son dossier ${ }^{66}$.

Cette règle est reprise dans les clauses d'amnistie conventionnelle ${ }^{67}$. Celles-ci varient quant à leur portée, selon la période requise avant que l'amnistie soit acquise, et selon qu'elles prévoient l'interruption du calcul du temps en cas de manquement ultérieur ${ }^{68}$. Entrent également en ligne de compte les termes utilisés dans la convention, dont la portée de la clause peut dépendre. 


\section{L'effet de la récidive}

Considérons le terme "récidive». Si le salarié a un comportement sans reproche, le problème ne se pose pas et le simple écoulement du temps suffit à l'acquisition de l'amnistie; si, au contraire, le salarié commet une seconde faute, l'hypothèse d'une différence de nature entre ces fautes peut susciter un débat. La récidive, en effet, consiste selon certains en la commission d'une seconde faute de même nature; ${ }^{69}$ par conséquent, il y a lieu de préciser si la condition consiste en la non-répétition de la première faute ou, plus strictement, en l'irréprochabilité du comportement durant la période convenue. Si l'expurgation du dossier est conçue comme une récompense d'une conduite méritoire, la seconde approche sera privilégiée. Mais s'il s'agit de sanctionner un manquement non imputable au salarié ${ }^{70}$ e.g. l'insuffisance professionnelle due à un mauvais état de santé, on peut se demander l'intérêt qu'il y a, une fois ce problème corrigé, à le jumeler à un autre, procédant d'une cause différente, qui se produirait subséquemment; ${ }^{71}$ naturellement, nous posons la question relativement à la légitimité de la conservation de la mention au dossier, plutôt que de l'utilité qu'elle pourrait avoir dans un arbitrage futur.

\section{L'effet de l'écoulement du temps}

Le second facteur est l'écoulement du temps. La longueur de la période est déterminée par les parties et il n'y a rien à dire sur ce sujet, sauf à noter que, plus courte est cette période, plus grande est l'interférence avec l'application de la progressivité des sanctions. Une fois la période définie, le mode de calcul du délai devient déterminant. En l'absence de précision, le délai se calcule en jours de calendrier, selon la méthode prévue au Code civil pour la prescription acquisitive ${ }^{72}$.

Le calcul du délai ne fait pas problème, sauf s'il échoit (malencontreusement ou intentionnellement) juste avant ou après la commission d'une faute subséquente. S'il échoit juste avant, ce n'est pas de chance pour le salarié et inversement. Par ailleurs, dans l'hypothèse où un grief concernant le manquement le plus récent est porté à l'arbitrage, le moment déterminant est celui où la cause du grief a pris naissance et non celui de l'ouverture de l'enquête; autrement, la négligence d'une partie (le syndicat agissant au nom du salarié) lui permettrait de priver l'autre d'un moyen de droit important i.e. de faire valoir les antécédents défavorables du réclamant ${ }^{73}$.

S'agissant d'un délai contractuel, à défaut de mode de calcul spécifié à l'entente, il faut donner aux termes leur sens commun: les jours, mois et 
années sont des jours, mois et années civils ou «de calendrier». Suivant une règle bien établie, le jour de l'échéance (jour ad quem) ne compte pas, c'està-dire que, si l'exécution d'une obligation est suspendue jusqu'à aujourd'hui, le créancier pourra la réclamer demain ${ }^{74}$.

Le calcul du délai pose aussi le problème de sa suspension ou de son interruption. Si le temps se compte en unités civiles, sans aucune restriction conventionnelle, comment un arbitre pourrait-il poser de telles restrictions, sans apporter des distinctions que la convention ne fait pas elle-même?

Ainsi, comment décider qu'une période de suspension, de mise à pied économique, de grève, de maladie, ${ }^{75}$ etc. suspendrait le cours du temps, alors que ces événements ne suspendent que l'exécution des obligations principales ${ }^{76}$ du contrat de travail - prestation de services et versement du salaire - sans pour autant rompre le lien contractuel? Il faudrait pour cela que le délai soit computé en jours ouvrables et ouvrés; or, une telle interprétation ne se justifie que si les parties se sont ainsi exprimées ${ }^{77}$. Par ailleurs, l'article 151.3 C.t. n'est ici d'aucune utilité, car il ne cọncerne que les délais fixés par le Code même. Nous ne l'invoquerons donc pas comme fondement d'un argument a contrario.

Enfin, parler de l'arrêt de l'écoulement du temps implique une analogie avec la suspension ou l'interruption de la prescription. La suspension de la prescription ne l'interrompt pas: elle arrête l'écoulement du temps sans que le délai ne soit reporté au point de départ. L'interruption, au contraire, anéantit le temps déjà écoulé au moment où elle a lieu ${ }^{78}$. Il est par conséquent important de faire la distinction au moment du calcul du délai. La récidive, dont nous avons déjà parlé, a un effet interruptif; la maladie, ou toute autre cause de suspension du contrat de travail, aurait seulement un effet suspensif. Comme une disposition expresse est essentielle pour donner naissance à l'une ou à l'autre, l'interprète devra s'en tenir au texte. Encore faut-il que les parties négociantes l'aient rédigé en connaissance de cause ${ }^{79}$.

\section{Portée de la clause d'amnistie}

La convention faisant la loi des parties, la portée de la clause d'amnistie se mesure à la lumière de l'intention des parties, telle qu'exprimée par les termes de l'accord.

Ce n'est qu'en cas d'obscurité des textes ou d'ambiguité de certaines dispositions que l'arbitre sera justifié de recourir aux règles d'interprétation des textes juridiques - lois ou contrats. En ce cas, l'article 1019 du Code civil pourra s'avérer utile: 
Dans le doute, le contrat s'interprète contre celui qui a stipulé, et en faveur de celui qui a contracté l'obligation.

La clause d'amnistie bénéficie au salarié; ${ }^{80}$ donc, entre deux interprétations également valables, l'arbitre choisira celle qui est la moins contraignante pour l'employeur. Il revient à l'agent négociateur de préciser l'étendue de l'avantage qu'il désire procurer aux salariés qu'il représente ${ }^{81}$.

Comme c'est à leur bénéfice qu'une telle clause est négociée, ne nous semble pas valide, en tant que règle générale, l'assertion de l'arbitre Courtemanche à l'effet que:

[...] la clause limitant la durée du passé disciplinaire subsistant au dossier est toujours à double tranchant. Elle impose d'apprécier le comportement d'un salarié dans le cadre de la durée convenue [...] et donc d'ignorer tant les incidents qui ont pu survenir auparavant que l'excellence du dossier antérieur $[\ldots]{ }^{82}$

(Italiques ajoutés)

Un texte clair serait nécessaire pour permettre d'inférer que le salarié ne serait pas admissible à invoquer la totalité de ses états de services, toujours en vertu de l'article 1019 du Code civil. Une clause d'amnistie a essentiellement pour objet de procurer un avantage au salarié; elle n'emporte renonciations à d'autres droits qu'en cas de termes exprès à cet effet. En particulier, le caractère synallagmatique de la convention collective ne permet pas une telle inférence, car la réciprocité ne s'attache qu'au tout et pas nécessairement à toute et chacune des clauses formant ce tout; il s'ensuit que dans une clause la réciprocité ne peut résulter que des termes de l'entente.

Sous réserve d'un texte exprès à l'effet contraire, un salarié pourrait renoncer ${ }^{83} \mathrm{au}$ bénéfice de l'amnistie s'il jugeait plus avantageux de présenter l'ensemble de son dossier au titre des circonstances dont doit tenir compte l'arbitre ${ }^{84}$. Il va sans dire que ce faisant, il laisserait le chemin libre à l'employeur qui pourrait faire la preuve de ses fautes passées ${ }^{85}$.

En l'absence de renonciation de la part du salarié, on a pensé que le devoir de tenir compte de toutes les circonstances d'une affaire permettrait à l'arbitre de passer outre à une clause d'amnistie, voire même l'y obligerait $^{86}$. L'affaire Wire Rope mettait en relief l'opposition qui peut exister entre une clause d'amnistie et l'article 98 (d) du Code du travail de la Colombie-Britannique qui se lit:

Sec. 98. For the purposes set out in section 92, an arbitration board has the authority necessary to provide a final and conclusive settlement of a dispute arising under a collective agreement, and without limiting the generality of the foregoing, has authority to

[...]

(d) determine that a dismissal or discipline is excessive in all circumstances of the case and substitute other measures that appear just and equitable. ${ }^{87}$ 
L'arbitre a décidé qu'une clause d'amnistie n'amputait pas la juridiction de l'arbitre en édictant que les fautes antérieures à telle période de temps ne sont pas pertinentes à l'évaluation de la sanction ${ }^{88}$. Plusieurs motifs peuvent rendre irrecevable la preuve d'un fait et l'accord des parties peut empêcher la mise en preuve du dossier disciplinaire d'un salarié, en tout ou en partie.

Au Québec, l'article 100.12 f) du Code du travail ${ }^{89}$ énonce pareillement que l'arbitre contrôle la décision disciplinaire «compte tenu de toutes les circonstances de l'affaire». Il faut ici aussi donner préséance à la volonté des parties exprimée dans une clause d'amnistie ${ }^{90}$.

Si la preuve d'une faute amnistiée n'est pas admise, il reste que l'arbitre en aura pris connaissance, au moins partiellement, à l'occasion du débat. Pour cette raison, dans une décision isolée, un arbitre américain a décidé que l'introduction en preuve de mesures disciplinaires à l'arbitrage en contravention d'une clause prohibitive justifiait l'annulation des sanctions ${ }^{91}$. Mais il faut se rappeler que le droit américain considère à maints égards que les exigences procédurales (due process) emportent le fond (substantive rights), ce qui n'est pas nécessairement le cas chez-nous.

\section{CONCLUSION}

L'amnistie des fautes disciplinaires mérite l'attention des parties patronales et syndicales à cause de la portée considérable qu'elle peut avoir sur le sort des griefs contestant une mesure disciplinaire. Cette attention est nécessaire aux deux niveaux de la négociation et de l'administration de la convention collective.

S'agissant de la négociation, il va sans dire que la teneur d'une clause d'expurgation du dossier discipliné doit être soigneusement examinée, notamment quant à la condition de non-récidive et à celle de la suspension du délai en certaines circonstances. De plus, la notion de dossier disciplinaire ne devrait pas être négligée car elle détermine dans une large mesure quels faits ou teneurs sont opposables au salarié en arbitrage.

Quant à l'amnistie avant sanction, celle qui résulte d'un geste de clémence consciente n'est pas de notre domaine. Par contre, celle qui est involontaire peut être identifiée par l'examen de la procédure disciplinaire suivie.

Le résultat de cet examen dépend de deux facteurs. D'abord, quelle est la procédure à suivre: la réponse dépend de la politique de l'employeur ou des règles de la convention collective, qu'il s'agisse de la teneur des mesures 
disciplinaires, du délai pour les imposer, de la progressivité des sanctions, etc. En outre, l'administration de cette politique ou de la convention pourra donner lieu à l'amnistie, selon les circonstances, si les règles établies ne sont pas suivies. À ce chapitre, l'initiative revient à l'employeur, tandis que le syndicat a le rôle de surveillance que lui confèrent d'une part la notion extensive du grief dans notre droit du travail et d'autre part la "propriété» $\mathrm{du}$ grief qui lui est dévolue.

Quel que soit le point de vue par lequel on aborde l'amnistie des fautes disciplinaires, il nous semble qu'elle implique des considérations d'un intérêt théorique et d'une portée pratique non négligeables. L'importance du concept d'amnistie est même appelé à s'accroître dans les années à venir dans la mesure où les exigences procédurales de l'exercice du pouvoir disciplinaire sont elles-mêmes appelées à se développer.

\section{NOTES}

1 Pour une conception semblable de l'amnistie, voir G.H. CAMERLYNCK, Gérard LYON-CAEN et Jean PÉLISSIER, Droit du travail, Précis Dalloz, treizième édition, Paris, Dalloz, 1986, $\mathrm{n}^{\circ} 360$. Le vocabulaire est emprunté au droit pénal dont le concept d'amnistie recouvre le pardon de la faute avant ou après condamnation: Gaston STEFANI et Georges LEVASSEUR, Procédure pénale, Précis Dalloz, dixième édition, Paris, Dalloz, 1977, $\mathrm{n}^{\circ} 121$. D'autre part, l'amnistie se distingue de la grâce en ce que cette dernière est une prérogative discrétionnaire échappant au contrôle juridictionnel des tribunaux: André DECOCQ, Droit pénal général, Collection U, Librairie Armand Colin, Paris, 1971, pp. 171 et seq.

On atteint ici les limites de l'analogie entre le droit pénal et le droit disciplinaire de l'entreprise. L'analogie n'en est pas toutefois sans utilité. Ainsi, le chef d'entreprise n'a certes pas le pouvoir de «gracier» discrétionnairement car dans la mesure où la discrétion exercée équivaut à discrimination, elle devient sujette au contrôle du juge ou de l'arbitre, selon le cas.

Les avantages et limites de l'application des concepts de droit criminel en droit disciplinaire sont clairement exposés dans Mark L. KAHN, Editor, Labor Arbitration. Perspectives and Problems, chapitre 6 par Sanford KADISH et al., Washington, Bureau of National Affairs, 1964, pp. 125-64.

Pour la France, voir: René de QUENAUDON, «L'exercice du pouvoir disciplinaire dans l'entreprise et écoulement du temps», (1984) Droit social 173, pp. 173-74, et notes (7) et seq.

2 Nous ne traiterons que de l'amnistie consentie par l'employeur. En France, la loi du 4 août 1981 a amnistié non seulement les employés de l'Administration - comme il était coutume de le faire pour inaugurer un septennat présidentiel - mais aussi les salariés de l'entreprise privée. Voir à ce propos: Jean SAVATIER, «L'amnistie des sanctions disciplinaires dans les entreprises», (1981) Droit social 609; Paul BOUAZIZ, «La loi d'amnistie et le pouvoir disciplinaire de l'employeur», (1981) Droit ouvrier 320; Jean-Marie GONNARD, «La loi $\mathrm{n}^{\circ}$ 81-736 du 4 avril 1981 portant amnistie», J.C.P. 1981, I. 3046; Jean-Charles GUILLARD et 
Philippe LAFARGE, «Amnistie civile, pardon ou profit», (1981) Gazette du Palais 443; Maxence RAYROUX, «L'amnistie en droit du travail», (1981) Gazette du Palais 446; Georges FLECHEUX et Philippe LAFARGE, “Observations sur l'amnistie septennale», (1981) Gazette du Palais 508; Jacques AUDINET, "La loi d'amnistie et le droit du travail» J.C.P. I982, I. 3059.

L'amnistie n'est pas étrangère à notre législation. Certaines de nos «lois de retour au travail» en recèlent des manifestations. Voir par exemple: Loi de 1987 sur le maintien des services ferroviaires, Bill C-87 adopté par la Chambre des Communes du Canada le 27 août 1987, articles $5(2) b, 14(2) b, 23(2) b$ et $32(2) b)$ interdisant aux entreprises visées de renvoyer un employé ou de lui imposer une autre mesure disciplinaire en raison de sa participation à la grève (comparer les textes anglais et français); Loi sur la reprise des travaux de construction, L.Q. 1986, c. 11, article 7 permettant au salarié «qui estime avoir été congédié sans cause juste et suffisante» entre le début de l'arrêt de travail et le moment de l'entrée en vigueur de la loi de soumettre une plainte en vertu de l'article 105 de la Loi sur les relations du travail dans l'industrie de la construction; Loi assurant aux usagers la reprise des services normaux de la Commission de transport de la Communauté urbaine de Montréal, L.Q. 1975, c. 56, article 3, deuxième alinéa («[a]ucune mesure disciplinaire ne doit, en raison de la grève actuellement en cours ou d'actes posés pendant cette grève, être exercée par la Commission contre un salarié [qui se conforme à l'ordre de retour au travail])»); Loi concernant l'industrie de la construction, L.Q. 1970 , c. 34, article 4 prohibant les mesures disciplinaires en raison d'une grève ou d'un lock-out durant la période visée par la loi. Dans une autre étude, il serait intéressant de comparer la portée de ces différents textes d'amnistie de même que les facteurs socio-politiques expliquant leur inclusion dans la loi aussi bien que leur variété.

Enfin, nous n'étudierons pas les ententes bilatérales de règlement des griefs, y compris l'annulation de mesures disciplinaires prises à l'occasion, en raison ou indépendamment de la grève, que contiennent les protocoles de retour au travail. Sur ce point, consulter: Claude D'AOUST et Louis LECLERC, Les protocoles de retour au travail: une analyse juridique, monographie $n^{\circ}$ 6, Ecole de relations industrielles, Université de Montréal, 1980, pp. 57-62.

Notre étude se limite à l'amnistie implicite ou explicite résultant de la conduite de l'employeur, action ou omission constituant un acte ou un fait juridique unilatéral. Nous examinons aussi l'effet de l'écoulement du temps sur la faute et la sanction tel que déterminé par la jurisprudence arbitrale et les clauses d'amnistie conventionnelles; ces dernières se distinguent des dispositions similaires des protocoles de retour au travail par la généralité de leur objet i.e. elles visent toutes les fautes de n'importe quel salarié en quelque moment qu'elles se produisent, si l'on ne retient que les modèles extrêmes.

3 Les effets de la faute et de sa sanction ne disparaissent que pour le futur. Pour cette raison, l'amnistie est inopérante lorsque la sanction consistait en un congédiement, sauf disposition spéciale au contraire.

Ainsi, le salaire perdu pendant une suspension ne sera pas remboursé. L'amnistie se distingue par là de l'annulation de la décision patronale qui a pour effet de remettre les parties en état.

4 En clair, nous retenons, pour les fins présentes, les mesures disciplinaire et non disciplinaire.

5 Sur la distinction entre les fautes professionnelle et disciplinaire, voir: Jean PÉLISSIER, «La définition des sanctions disciplinaires», (1983) Droit social 645, pp. 647-48; de QUENAUDON, loc. cit., pp. 174-75; Jean SAVATIER, «Droit du travail: jurisprudence commentée», (1983) Droit social 634, pp. 643-44.

6 Sur la révision du contrat en général, voir: Jacques FLOUR et Jean-Luc AUBERT, Les obligations, volume I, Paris, Librairie Armand Colin, 1975, pp. 311 et seq.; Alain GHOZI, La modification de l'obligation par la volonté des parties, Bibliothèque de droit privé, tome XLXVI, Paris, L.G.D.J., 1980. 
Sur la modification unilatérale du contrat de travail, voir: Bernard TEYSSIÉ, «La modification du contrat de travail, instrument de gestion de l'entreprise", (1986) Droit social 852. Dans l'intérêt de l'entreprise et sous la pression des impératifs économiques, l'employeur peut modifier unilatéralement le contenu obligationnel du contrat de travail, l'acquiescement tacite du salarié emportant novation.

Il n'y a pas de raison que ce mécanisme ne joue pas dans les deux sens. Si l'employeur peut retirer des «avantages acquis» au salarié avec son accord tacite (par exemple, une diminution du taux de salaire), le salarié de son côté pourrait diminuer la qualité ou la quantité de sa prestation avec la bénédiction du patron. Dans les deux hypothèses, l'intensité de l'obligation est modifiée et non sa nature. En d'autres termes, il n'y a pas novation au sens technique, laquelle ne se présume pas et nécessite l'extinction de l'obligation première. Voir G.H. CAMELYNCK dans Répertoire de droit du travail, Encyclopédie Dalloz, $\mathrm{V}^{\circ}$ contrat de travail (modification), nos 36 et seq. Pour s'opposer à la modification unilatérale, l'employeur devra aviser le salarié de son motif d'insatisfaction.

Cette obligation de l'employeur de signaler au salarié ses déficiences comme préalable de la cause juste de renvoi a été reconnue en jurisprudence arbitrale (normes québécoises et canadiennes du travail): Gilles TRUDEAU, Statutory protection against unjust dismissal for unorganized workers, doctoral dissertation, School of Law, Harvard University, 1985, p. 168 et les sentences arbitrales mentionnées en note infrapaginale. Ce principe a été reconnu implicitement et a contrario dans Guay c. Agropur, Coopérative agro-alimentaire, [1986] T.A. 78 (en l'absence d'un salarié handicapé, l'employeur procède à des changements technologiques de sorte qu'au retour le salarié a une productivité moindre qu'auparavant alors que, comme d'autres, il jouissait d'un «traitement de faveur", p. 81). Cependant, s'agissant d'une maladie (alcoolisme), le fait que l'employeur ait appliqué une sanction moindre que celle prévue par le règlement intérieur sera neutralisé par le facteur aggravant de la «récidive», ultérieurement: $L a$ Fraternité des chauffeurs d'autobus, opérateurs de métro, services connexes de la CTCUM et CTCUM, (1980) S.A.G. 391.

7 Voir l'énoncé très clair de la nécessité de signaler au salarié son insuffisance professionnelle et de le faire diligemment: Re McKellar General Hospital and Canadian Union of Public Employees, Local 1409, (1986) 24 L.A.C. (3d) 69; Re Denison Mines Ltd. and United Steel Workers, (1983) 12 L.A.C. (3d) 364; Re Edith Cavell Private Hospital and Hospital Employees' Union, Local 180, (1982) 6 L.A.C. (3d) 229; Automatic Electric (Canada) Ltd. and International Union of Electrical, Radio \& Machine Workers, Local 526, décision non rapportée de M. G. Picher, 1978 et citée dans Re Denison Mines, supra, aux pages 367 et 370-71; Re International Union of Electrical Workers and J.A. Wilson Lighting and Display Ltd., (1958) $8 \mathrm{~L}$.A.C. 156. Dans ce dernier cas, il a été décidé que l'employeur ne peut rétrograder un salarié pour incompétence - dans les arrêts précédents, il s'agissait de renvois — s'il ne lui en a jamais fait reproche depuis son embauchage.

Tous ces arrêts reconnaissent qu'il y a, en certaines circonstances, obligation d'informer le salarié de ce qu'on lui reproche avant de prendre une mesure pour pallier un manquement non disciplinaire («non-culpable», «blameless», «non disciplinary» cause).

Le principe de progressivité des sanctions (répressives) est à distinguer de la manifestation d'une opposition à la modification unilatérale, quoiqu'involontaire, des termes du contrat de travail. Mais un arbitre a, dans un arrêt isolé, fait une analogie entre la progressivité des sanctions et la révision du contrat de travail (les accommodements que devrait exiger l'entreprise en difficulté au lieu d'attendre que la situation soit à ce point détériorée qu'elle nécessite le recours au licenciement, mesure ultime): De Melo c. The Dog Studio, [1984] T.A. 460, à la p. 463, deuxième colonne.

8 Art. 1067 C.c.: l'interpellation en justice est elle-même une forme de mise en demeure.

9 Nous faisons abstraction des garanties procédurales qui s'imposent à l'employeur. Voir Claude D'AOUST et Louise DUBE, «Le devoir d'équité procédurale de l'employeur privé»,, (1987) Revue du Barreau 667. 
10 Si une sanction a déjà été imposée, la situation s'analyse de deux façons. Selon la première explication, la décision patronale serait tenue pour non définitive tant que le grief n'est pas porté à l'arbitrage. Si, au contraire, cette décision est considérée définitive, bien que sujette à révision, soit par entente entre les parties, soit par décision d'un tiers-arbitre, l'hypothèse présentement examinée se rangera dans la classification de l'amnistie après sanction, en cas de règlement à l'amiable (techniquement appelé «transaction»).

11 Notre Code du travail ne mentionne pas la procédure interne de grief sous ce vocable.

Les articles 100.0.2 et 100.3 traitent du règlement à l'amiable. Si les parties y donnent suite, l'arbitre le constate et en donne acte. Si l'une d'elles refuse de s'y conformer - et l'amnistie non respectée n'est qu'une des hypothèses possibles - l'autre pourra la lui opposer à l'arbitrage, en faisant la preuve de la transaction et à condition que la "règle des trois identités» soit respectée (identité de parties, d'objet et de cause). Si la faute amnistiée est plus tard reprochée au salarié à l'occasion de la sanction d'une faute ultérieure, l'arbitre pourra en disposer au titre de l'admissibilité de la preuve ou de sa pertinence (une preuve qui n'a aucune valeur probante n'est pas pertinente).

Par ailleurs, l'amnistie, acte unilatéral, devient irrévocable dès qu'elle est notifiée a l'autre partie et acceptée par elle: Yves SEILLAN, «L'acte abdicatif», (1966) Revue trimestrielle de droit civil $686, \mathrm{n}^{\circ} 50, \mathrm{p}$. 707. Une analyse plus traditionnelle serait conduite en termes de renonciation à un droit (de créance). Mais la remise de dette est nécessairement bilatérale, tandis que dans l'hypothèse examinée présentement, la renonciation expresse à exercer une faculté (non plus un droit de créance) peut se faire par le simple jeu de la volonté du titulaire. La seule question intéressante est alors de savoir quand et à quelle condition il ne lui est plus loisible de revenir sur sa décision.

12 La faculté d'alléger la peine après l'expiration du délai imparti pour la sanction ne paraît pas douteuse, si le délai a été initialement respecté, qu'il s'agisse d'un acte de clémence ou d'apaisement ou encore d'une rectification (par exemple si les faits fautifs se révèlent moins graves qu'un premier examen ne l'avait d'abord indiqué) ou même d'une concession en vue d'éviter un litige.

Le pouvoir disciplinaire s'exerce à la discrétion de l'employeur, dans les limites de l'ordre public, des bonnes moeurs, de la loi et du contenu obligationnel du contrat individuel de travail et de la convention collective. Voir: Robert P. GAGNON, Louis LeBEL et Pierre VERGE, Droit du travail, Québec, P.U.L., 1987, pp. 462 et seq. Dans cette veine, on peut arguer que la prescription de la faculté de punir s'interprète restrictivement et ne couvrirait pas, sauf mention expresse, la faculté de pardonner. En principe, un «acte de pure faculté» ne se prescrit pas, aux termes de l'article 2196 C.c. et si l'on peut admettre qu'une convention collective ou la jurisprudence dérogent à cette règle de droit commun, encore faudrait-il admettre que telle dérogation doive s'interpréter restrictivement.

En tout cas, l'adoucissement de la sanction dans le cadre de la procédure de règlement des griefs est, en principe, indiscutablement valide. Cela est tellement vrai qu'une réduction «négociée» de la sanction en procédure interne de grief empêche le syndicat de contester la sanction initiale: Syndicat international des travailleurs unis de l'automobile, de l'aéronautique, de l'astronautique et des instruments aratoires, Section locale 1581, c. Atlas \& Body Hoist Inc., (1974) S.A.G. 506. Si l'employeur modifie une suspension en un avis disciplinaire, au stade de l'arbitrage, sans l'accord du syndicat, la juridiction de l'arbitre n'est pas affectée: $S$. Coorsh \& Sons Limited c. Le Syndicat des travailleurs canadiens de l'alimentation et d'autres industries et Claire Lescarbeau, (1979) S.A.G. 645 et la jurisprudence citée.

En France, la Cour de cassation a répondu par l'affirmative à la question ici soulevée: Agneray et al. c. Société des transports départementaux de la Marne, Chambre sociale, 27 février 1985, (1985) Recueil Dalloz-Sirey, I.R. 442 (le règlement intérieur ne limitait pas la faculté de pardonner; par ailleurs, une telle mesure favorisait le salarié). La décision de la Cour 
de cassation montre, par ses motifs, que la faculté d'amnistier n'est pas un cas particulier d'un droit plus général de modifier unilatéralement une sanction (à l'intérieur des délais ou après leur expiration). L'employeur, en effet, ne peut alourdir une sanction déjà imposée, même à l'intérieur des délais. La règle de la double sanction l'en empêche. Seule la découverte de faits inconnus au moment de l'imposition de la sanction, ou qu'une recherche diligente ne lui aurait pas permis de découvrir, pourrait justifier un tel ajustement.

Par contre, une mesure non disciplinaire (e.g. une observation verbale) pourrait être suivie d'une mesure disciplinaire, à l'intérieur des délais impartis. À l'inverse, après écoulement du délai, il n'est pas permis d'effectuer ce changement: Re Corporation of the Borough of North York and Canadian Union of Public Employees, Local 289, (1979) 20 L.A.C. (2d) 289, à la p. 296; Re Oxford Pendaflex Canada Ltd. and Printing Specialties \& Paper Products Union, Local 466, (1977) 14 L.A.C. (2d) 104 (lorsqu'un représentant de l'employeur, apparemment autorisé à la faire, impose une sanction immédiatement après la commission d'une faute, son supérieur hiérarchique ne peut lui substituer une sanction plus lourde, même si un dossier antérieur chargé justifie cette dernière); Re Long Manufacturing Division, Borg-Warner (Canada) Ltd. and United Automobile Workers, Local 1256, (1976) 11 L.A.C. (Ed) 395 (ce sont les circonstances qui déterminent si la première mesure prise par l'employeur est définitive et donc si une autre sanction peut être prise par la suite); Re Galt-Brantford Malleable Ltd. and International Molders and Allied Workers, Local 29, (1974) 6 L.A.C. (2d) 302 (lorsque la convention collective prévoit qu'un simple avis verbal est une mesure disciplinaire, l'employeur ne peut en changer par la suite); Re United Steelworkers, Local 5615 and Rio Algoma Mines Ltd., (1962) 12 L.A.C. 194 (un supérieur hiérarchique n'est pas habilité à réviser une réprimande écrite imposée par un contremaître); Re International Union of Electrical Workers and J.A. Wilson Lighting \& Display Ltd., (1958) 8 L.A.C. 156 (une rétrogradation suivant une suspension est une mesure disciplinaire - par opposition à une mesure administrative fondée sur l'opinion que l'acte reproché au salarié révélait son incompétence - parce que l'employeur ne lui avait fait aucun reproche quant à la qualité de son travail depuis qu'il occupait ses fonctions, p. 160); Re United Electrical, Radio \& Machine Workers, Local 520 and A.H. Tallman Bronze Co. Ltd., (1957) 7 L.A.C. 253 (l'un des trois arrêts de principe en la matière, avec les deux mentionnés ci-après); Re United Automobile Workers, Local 673 and De Havilland Aircraft Ltd., (1957) 7 L.A.C. 61 (une sanction imposée pour réprimer la répétition d'une faute s'ajoute validement à la sanction visant la commission de cette faute elle-même); United Automobile, Aircraft and Agricultural Implement Workers of America (UAW-CIO) in re The Fruehauf Trailer Company of Canada Limited, (1951) 3 L.A.C. 347 (lorsqu'une personne en autorité - ici le directeur du personnel - informe un salarié qu'un incident ne donnera lieu à aucune mesure disciplinaire si telle condition est remplie - en ce cas-ci la présentation d'excuses au contremaître injurié à l'occasion d'une discussion - un cadre ne peut par la suite imposer une sanction si les termes de l'arrangement à l'amiable ont été respectés).

Comparer, pour le Québec: Le Syndicat professionnel des policiers de Beauport Inc. c. La Ville de Beauport, (1981) S.A.G. 2045; Association des policiers-pompiers de Ville de St-Georges-Est c. Ville de St-Georges-Est, [1986] T.A. 653. L'autonomie municipale étant entamée par la juridiction de la Commission de Police en matière disciplinaire, la validité et la portée des appréciations ou promesses des supérieurs hiérarchiques en sont réduites d'autant.

L'opposabilité du manquement amnistié dans un litige subséquent sera examinée plus loin.

Enfin, pour qu'il y ait amnistie, la mesure initialement prise doit être définitive. Par conséquent, la suspension pour enquête n'empêche pas qu'une mesure définitive, par exemple le renvoi, soit ultérieurement arrêtée.

13 Observons que dans un grief disciplinaire la preuve d'éléments non disciplinaires n'est pas nécessairement exclue. Ainsi, un arbitre a noté que si une promotion est accordée à un 
salarié, les fautes disciplinaires qui l'ont précédée pourraient ne plus lui être opposables dans un grief subséquent: La Caisse populaire de St-Anaclet $\mathrm{c}$. Lise Blanchette, décision selon l'art. $124 L N T$, non rapportée, le 26 avril 1988, M. Morin, arbitre, p. 23.

14 L'article 100.12 f) C.t. reconnaît implicitement la légalité et le caractère impératif d'une liste complète, sans pour autant exclure une liste partielle.

Le législateur français a procédé inversement. L'article L. 122-40 du Code du travail Dispose:

Constitue une sanction toute mesure, autre que les observations verbales, prise par l'employeur à la suite d'un agissement du salarié considéré par lui comme fautif [...].

Il résulte de ce procédé que l'employeur peut imposer toute sanction disciplinaire, même celle que ne prévoit pas le règlement d'entreprise: voir Code du travail, édition réalisée par Bernard TEYSSIÉ, Paris, Librairies techniques, 1987, p. 109, note 2.

15 A contrario, une mesure patronale n'est considérée comme mesure disciplinaire que s'il en est fait mention dans le dossier disciplinaire. En ce sens, en droit français: Agneray et al. c. Société des Transports départementaux de la Marne, Cour de cassation (Chambre sociale), 27 février 1985, Recueil Dalloz-Sirey, I.R. 442 et commentaire de Philippe Langlois; British Columbia Hydro and Power Authority v. The International Brotherhood of Electrical Workers (Gabb), Canada Labour Views Reports, April 8, 1985, pp. 1-2.

16 Les salariés peuvent se protéger contre cette éventualité en inscrivant à la convention collective la définition du dossier du salarié et son contenu, donc le dossier admissible en preuve à l'arbitrage. Voir par exemple: The Government of the Province of Alberta and The Alberta Union of Provincial Employees, 8 C.L.A.S. 9, No. 08:0056, p. 3. Le Canadian Labour Arbitration Summaries est publié par Canada Law Book Co.

Une décision ontarienne récente illustre à quel point la notion de «dossier du salarié» est loin d'être univoque. En l'espèce, la convention collective comportait une clause d'amnistie. Lorsqu'une faute était amnistiée, toute trace matérielle en était effacée du dossier conservé au niveau du département. Mais la direction des ressources humaines gardait aussi un dossier général sur chaque salarié, relatant son «histoire». Les mesures disciplinaires y demeuraient notées indéfiniment, et en particulier au-delà de la période d'amnistie. L'arbitre reconnut à l'employeur le droit de conserver un pareil dossier ou tout autre dossier qu'il jugerait utile, à condition que le cadre participant à l'imposition d'une mesure disciplinaire n'en prenne pas connaissance. Le cadre-conseil du service des ressources humaines devrait donc, à l'avenir, consulter un autre dossier que celui que conservait son service.

Il n'en reste pas moins que selon cette décision, fort bien construite par ailleurs et à laquelle notre résumé ne saurait rendre justice, des informations relatives au passé disciplinaire d'un salarié peuvent être conservées dans divers dossiers et à diverses fins. Le stockage informatique de données offre à cet égard des possibilités virtuellement illimitées quant à la nature des données conservées, à la période couverte, etc. La distinction entre les mesures disciplinaires et non disciplinaires n'est pas sans rapport avec cette multiplication de renseignements car si les mesures disciplinaires sont, en pratique, divisibles en un nombre relativement limité de sous-catégories (e.g. l'avertissement oral ou écrit, la suspension, le congédiement étant les plus connus), on ne peut, à notre avis en dire autant des mesures dites «administratives».

Voir: Denison Mines Limited and United Steelworkers of America, 9 C.L.A.S. No. 09:0186. Cette décision de Harry Freedman soulève beaucoup d'autres questions. L'une des plus intéressantes est celle - non résolue - de l'effet relatif d'une clause d'amnistie à l'égard des tiers, par exemple un employeur faisant une vérification en relation avec une demande d'emploi.

17 Par exemple: Re City of Corner-Brook and C.U.P.E., Local 706, (1987) 27 L.A.C. (3d) 439, à la p. 443. Voir cependant: La Bonne Humeur (1975) Limitée c. Union des employés du commerce, Section locale 500, (1981) S.A.G. 1225, 1237. 
18 Id.

19 Citipark, Division de Silicom Inc. c. Union des employés de service, Local 298, [1984] T.A. 196 , à la p. 200.

20 Hilton International Québec c. Syndicat des travailleurs du Hilton Québec, D.T.E. $\mathrm{n}^{\circ} 86 \mathrm{~T}-531$, pp. 7,15 et 16.

De même, la prolongation unilatérale de la période d'essai n'est pas un avertissement disciplinaire: Re Old Dutch Foods Limited and UFCW, Local $373 A, 8$ C.L.A.S. 61, No. 08:0371. Ne sont pas non plus considérées comme mesure disciplinaire des plaintes de la clientèle de l'entreprise, même si, en ce cas, la convention collective autorise l'employeur à muter le salarié et donne à ce dernier un droit de déplacement (bumping): Sécurité et Protection Sec-Pro Inc. c. Union des agents de sécurité du Québec, Métallurgistes Unis d'Amérique, Local 8922, 8 C.L.A.S. 7, No. 08:0043, pp. 5 et 7, en obiter.

21 Syndicat de la rédaction du journal Le Devoir (CSN) c. Imprimerie populaire Ltée, D.T.E. $\mathrm{N}^{\circ} 85 \mathrm{~T}-443$, p. 11.

22 La Presse c. Syndicat des employés de bureaux de journaux (Région de Montréal), D.T.E. $\mathrm{n}^{\circ} 84 \mathrm{~T}-367$, p. 8. Cette sentence est citée et approuvée dans Shell Canada Ltée c. Travailleurs unis du pétrole du Canada, Local l, [1988] T.A. 188.

23 Ibid., p. 8.

24 En voici quelques exemples: Re Mount Sinai Hospital and Nurses' Association of Mount Sinai Hospital, (1973) 13 L.A.C. (2d) 103, particulièrement aux pages 106 et seq. (note faisant suite à une évaluation de rendement); Re Owen Sound General and Marine Hospital and Ontario Nurses's Association, (1977) 16 L.A.C. (2d) 11, particulièrement aux pages 18 et seq. (une entrevue entre une infirmière fautive et la personne responsable du nursing équivaut à un avertissement oral, le caractère professionnel de la tâche expliquant l'absence de formalité); Re Toronto East General and Orthopaedic Hospital Inc. and Ontario Public Service Employees Union, Local 576, (1979) 21 L.A.C. (2d) 296, pp. 303-8 (s'agissant d'un technicien employé dans une banque de sang, une procédure informelle équivaut à une procédure disciplinaire si les buts et fonctions du principe de progressivité sont respectés et ce, même si les avis oraux n'ont pas été notés ni copie des avis écrits déposée au dossier); Re Bay Forest Product Ltd. and International Woodworkers of America, Local 1-217, (1979) 21 L.A.C. (2d) 212, p. 215 (l'employeur qui fait des commentaires et donne des conseils doit en aviser le salarié s'il veut donner un caractère disciplinaire à ses remarques et le prévenir que mention en sera faite à son dossier, sans quoi les faits reprochés à cette occasion seront irrecevables en preuve); Duval Corp. (1964) 43 LA 102, à la page 106 (l'usage dans l'entreprise peut servir de guide pour déterminer quel type d'avertissement constitue une sanction disciplinaire, de même que l'acquiescement à sa mise en preuve); Liquid Carbonic Inc. et Syndicat des Métallos [sic], le 26 juin 1987, Léopold Lavoie, décision non publiée, p. 11 (la convention collective énumérant limitativement les mesures disciplinaires, si la preuve ne révèle l'imposition d'aucune d'entre elles, le dossier disciplinaire est réputé intact); Collège Beaubois c. Association des enseignants de Beaubois, (1981) S.A.G. 420, 427 (un rapport d'évaluation équivaut à un avis disciplinaire s'il comporte un élément de blâme et une incitation à un changement d'attitude); Shell Limitée c. Travailleurs unis du pétrole du Canada, Local 1, 8 C.L.A.S. 92, No. 08:0543 (un mémorandum déposé au dossier d'un salarié a valeur de mesure disciplinaire).

25 Laurentian Hospital and CUPE, Local 161, 5 C.L.A.S. 62, No. 05-0351, pp. 12-13.

26 Parce qu'il peut lui être opposé plus tard, cet avis doit pouvoir être contesté quand les faits qui y ont donné lieu sont récents, ajoute l'arbitre. Il suit de là que chaque fois qu'une mesure donne matière à grief, cette mesure doit être prise sans délai, de manière à assurer au salarié la possibilité de faire valoir tous ses moyens (ou, suivant une formule répandue, la possibilité de présenter une défense pleine et entière). Mais cet aspect de la question nous ramène directement à l'amnistie par prescription, c'est-à-dire résultant du défaut de sévir dans un délai 
raisonnable. Voir par exemple: Re Corporation of the Borough of North York and Canadian Union of Public Employees, Local 373, (1979) 20 L.A.C. (3rd) 288, pp. 290-1; Re: Air Canada and Canadian Airline Employees Association, (1982) 4 L.A.C. (3d) 68, p. 72; Domtar Inc. et Syndicat canadien des travailleurs du papier, Section locale 1492, D.T.E. $n^{\circ} 87 T-798$, p. 9.

27 Comme nous l'avons déjà signalé, il s'agit plutôt d'une faculté que d'un droit. Soutenir qu'elle peut s'éteindre par prescription équivaut à reconnaître que ce n'est pas une «pure» faculté, aux termes de l'article 2196 C.c.; à notre avis elle est limitée par les garanties de procédure destinées à protéger les droits du salarié faisant l'objet d'une sanction.

Comparer l'article 11, paragraphes a) et b) de la Loi de 1982 sur le Canada, 1982, c. 11 (R.-U.). Ces dispositions ne s'appliquent pas en droit disciplinaire. Voir: Henri BRUN, Chartes des droits de la personne, Collection «Alter ego», Montréal, Wilson et Lafleur, 1988, p. 136. Voir aussi l'article 32.1 de la Charte des droits et libertés de la personne, L.R.Q., c. C-12, ibid., p. 306; Trimm v. Durham Regional Police Force, [1987] 2 R.C.S. 582, 29 Admin. L.R. 106 et la jurisprudence citée.

Par ailleurs, la doctrine française de droit pénal parle de prescription de l'action publique, que l'on compare à la prescription de l'action civile: STEFANI et LEVASSEUR, op. cit., $n^{\circ} 127$. Cette prescription est une amnistie intervenue avant le jugement de condamnation: $i d$, $\mathrm{n}^{\circ} 121$.

28 Syndicat international des travailleurs, Lacal 333 c. La Compagnie Sucre Atlantic Ltée et al., [1981] C.A. 416, arrêt commenté par Thomas M. DAVIS, «La prescription en vertu de l'article 71 du Code du travail: un moyen de droit québécois que l'on peut soulever à la place des «laches», (1987) Revue du Barreau 585.

29 Le pouvoir disciplinaire existait avant l'avènement de la négociation collective, historiquement et existe dans la phase pré-syndicale d'une nouvelle entreprise. En ce sens, les clauses de reconnaissance du pouvoir de direction, dont le pouvoir disciplinaire est une branche, ne créent pas ce pouvoir: la convention collective en préserve l'existence et en encadre l'exercice.

Sur ce point, voir, par analogie: Fernand MORIN et Jules BRIĖRE, «Le droit de former une association de salariés est-il un droit résultant du Code du travail?», (1968) 23 Relations industrielles 501. Paraphrasant ce titre nous dirions: «Le pouvoir disciplinaire résulte-t-il (naît-il) de la convention collectiven? Sur le point précis de la source du pouvoir disciplinaire, voir: Le Syndicat professionnel des policiers de Beauport Inc. c. La Ville de Beauport, (1980) S.A.G. 2045 (il faudrait une disposition expresse à l'effet que le pouvoir disciplinaire naît de la convention collective pour que l'article 71 du Code du travail s'y applique); Le Syndicat des professeurs du Séminaire St-François de Cap-Rouge c. La Corporation du Séminaire St-François, (1981) S.A.G. 171; La Commission de transport de la Communauté urbaine de Montréal c. La Fraternité des chauffeurs d'autobus, opérateurs de métro et employés des services connexes de la CTCUM, (1981) S.A.G. 561, p. 567 (l'article 71 C.t. ne s'applique pas et, en l'espèce, les délais d'imposition de la mesure disciplinaire ne sont pas déraisonnables); Les Métallurgistes Unis d'Amérique, Unité 6932 c. Les Industries de Métaux Noranda Limitée, (1981) S.A.G. 1709.

30 Reproduite dans l'arrêt Corps Canadien des Commissionnaires, Division Montréal et Union des Agents de sécurité du Québec, Métallurgistes Unis d'Amérique, 8922, F.T.Q., [1987] T.A. 719, à la p. 720.

31 On pense ici à l'opposition entre procedural et substantive rights en droit administratif.. Voir: David Phillip JONES and Anne S. de VILLARS, Principles of Administrative Law, Toronto, Carswell, 1985, pp. 190-191; David J. MULLAN, «Natural Justice and Fairness Substance as well as Procedural Standards for the Review of Administrative Decision-Making?», (1982) 27 McGill Law Journal 250.

En droit administratif, il s'agit de déterminer si, ayant le pouvoir de sanctionner le non-respect des exigences procédurales, les tribunaux de droit commun ont aussi celui de toucher le fond (merit). Autre est la question de savoir si l'annulation d'une décision pour défaut 
de procédure entraîne la perte du pouvoir de sanctionner à nouveau le manquement que l'employeur avait voulu réprimer. Voir: Claude D'AOUST, «Effets de l'annulation d'un avis de congédiement par l'arbitre», (1984) 39 Relations industrielles 365.

Ici, nous ne discutons pas tellement d'un délai de procédure que d'un délai au-delà duquel l'employeur ne peut sanctionner. L'analogie se fait done plus naturellement avec la perte d'un droit qu'avec la prescription d'un droit d'action ou encore avec la perte de juridiction d'un tribunal auquel la loi impartit un délai pour agir. (On peut aussi raisonner en termes d'acquisition du pardon par le salarié; dans ces conditions, seul le caractère continu du manquement permettrait à l'employeur de remédier à sa négligence ou de mettre fin à sa clémence).

32 Re Teamsters Union Local 800 \& Reimer Express Lines Ltd., (1958) 8 L.A.C. 341 (renvoi survenu un an après que l'employeur eût découvert une fausse déclaration à l'embauche); Re Corporation of the Borough of North York and Canadian Union of Public Employees, Local 373, (1979) 20 L.A.C. (2d) 289 (négligence à faire une certaine tâche sur une période de douze mois et congédiement quelque huit mois après la fin de cette période). En jurisprudence québécoise, voir: Association nationale catholique des commis quincaillers et matériaux de construction de Québec Inc. c. Gauthier Ltée, (1970) S.A.G. 92, 95 (utilisation, sans permission, d'un camion sans intervention de l'employeur); Corporation de la Ville de St-Rémi c. Fraternité des policiers de St-Rémi, (1981) S.A.G. 1755 (un policier soupçonné d'abus de pouvoir à l'endroit d'un citoyen doit être puni ou exonéré dans un délai raisonnable depuis la survenance des faits, même si le citoyen est lui-même poursuivi au criminel pour entrave au travail du policier - application de la «théorie du délai raisonnable» et non-applicabilité de l'article 71 C.t.); C.U.M. c. Fraternité des policiers de la C.U.M., [1986] T.A. 442, p. 447 (le cas du policier soupçonné de voies de fait doit être soumis au processus disciplinaire avec célérité et sans attendre la conclusion de son procès criminel); Association des policierspompiers de Ville de St-Georges-Est c. Ville de St-Georges-Est, [1986] T.A. 653 (un délai de deux ans suivant un acte de brutalité d'un policier rend nulle la mesure disciplinaire adoptée et l'employeur n'était pas justifié d'attendre les recommandations devant suivre l'enquête de la Commission de Police du Québec). Adde: Ville de Joliette c. Fraternité des policiers de Joliette, D.T.E. ${ }^{\circ} 88 \mathrm{~T}-746$, p. 15 et seq.

A toutefois été jugée sans conséquence une attente de deux semaines avant d'ouvrir l'enquête sur un incident qui plus tard a, avec d'autres manquements, amené l'employeur à congédier le salarié: Great Atlantic \& Pacific Tea Co. Inc., 71 LA 805, à la p. 807. De même, un intervalle de deux semaines entre la commission de la faute et l'imposition de la sanction a été jugé raisonnable, vu que le salarié n'en avait pas subi de préjudice, notamment au plan de ses moyens de défense: CTCUQ c. Syndicat des employés de garage d'autobus de l'Est du Québec, [1985] T.A. 198.

Enfin, la connaissance des faits est acquise à la fin de l'enquête qu'institue l'employeur et, si la convention prévoit un délai fixe pour l'imposition d'une sanction, la computation commence à ce moment-là: La Compagnie minière Québec Cartier c. Les Métallurgistes Unis d'Amérique, Local 7401 et 7401 Force protectrice, (1976) S.A.G. 548, pp. 550-51; Citipark, Division de Citicom Inc. c. Union des employés de service, Local 298, [1984] T.A. 196, p. 200. Voir aussi: Syndicat des employés de bureau et de l'information (CSN) c. Progrès du Saguenay, D.T.E. $\mathrm{n}^{\circ}$ 85T-833, p. 10; Ciment indépendant Inc., Division Demix c. L'Association des employés de Demix, [1986] T.A. 260, p. 268.

33 Re Brunswick Bottling Ltd. and Retail, Wholesale and Department Stores Union, Local 1065, (1985) 16 L.A.C. (3d) 249.

34 Domtar Inc. c. Syndicat canadien des travailleurs de papier, section locale 1492, D.T.E. $\mathrm{n}^{\circ}$ 87T-798. L'arbitre fait une analogie avec la théorie des laches qui ne tient pas compte de la distinction entre la perte d'un droit et celle d'un recours; or, les laches s'appliquent à celle-ci, non à la première. L'analogie avec la poursuite criminelle qui doit intervenir 
promptement suivant diverses chartes des droits de la personne, est plus convaincante, par son pouvoir évocateur sinon par sa logique interne.

Voir aussi les arrêts CUM et Ville de St-Georges-Est, supra.

35 Re National Groceries Co. Ltd. and Teamsters Union, Local 419, (1984) 11 L.A.C. (3d) 193. Dans cette affaire, la convention collective établissait le délai pour agir; le raisonnement de l'arbitre s'appliquerait donc a fortiori en l'absence de clause, vu qu'un délai conventionnel est parfois considéré de rigueur.

Voir, au même effet; In Re Van World - Stevens Company and Graphic Arts International Union, Local 1B, 79 LA 645, p. 649 (l'employeur avait tardé à signifier son congé au salarié sous prétexte de l'hospitalisation de ce dernier).

36 Inland Tool \& Mfg. Inc., 65 LA 1203 (1965), approuvée dans In Re Associated Grocers of Colorado Inc. and International Brotherhood or Teamsters, Chauffeurs, Warehousemen and Helpers of America, Local 435, 82 LA 414, p. 419 (délai de quatre mois entre la commission de la faute et la suspension pour enquête et délai additionnel de deux mois avant le renvoi). Dans un cas où le salarié avait été hospitalisé après la commission de sa faute et où l'employeur avait aussi attendu la fin du traitement pour congédier le salarié, l'arbitre ne se prononce pas sur le point parce que la sanction avait été prişe sans que le salarié ne soit entendu: In Re McCartney's Inc. and United Food and Commercial Workers International Union, Local 76, 84 LA 799, p. 804.

De toute manière, ni l'entretien avec le salarié, ni l'imposition de la sanction ne doivent nécessairement avoir lieu le jour de la commission de la faute, l'employeur disposant d'un délai raisonnable pour s'enquérir des faits et jauger l'opportunité de la sanction: In Re Intermountain Rural Electric Association and International Brotherhood of Electrical Workers, Local 111, 86 LA 540.

37 Independent Bus Garage Company Inc. and Amalgamated Transit Union, Local 1156, 69-1 ARB parag. 8160; Re North York General Hospital and Canadian Union of General Employees, (1974) 15 L.A.C. (2nd) 45, p. 47.

L'accumulation des fautes au dossier, à l'insu du salarié, viole le principe de progressivité des sanctions. Ce principe a été reconnu de longue date par notre jurisprudence arbitrale et il n'est pas nécessaire de citer des arrêts à cet effet. Ajoutons seulement un cas particulier: si l'employeur surveille le salarié, sans que celui-ci ne commette de faute (contrairement à l'hypothèse précédente) il n'y a pas cause à renvoi a la première occasion, surtout lorsqu'un long intervalle sépare cette faute du manquement précédent qui était à l'origine de la surveillance. Voir: Bérubé c. Marcel E. Savard Inc., Cour provinciale, district de Mingan, dossier $\mathrm{n}^{\circ}$ 650-02-000262-855, le 18 septembre 1987, p. 5; Re SKF Manufacturing of Canada Ltd. and International Association of Machinists, Local 901, (1975) L.A.C. (2nd) 139, p. 140.

La question peut aussi être examinée sous l'angle de l'équité procédurale. Comme le disait un arbitre américain:

«Secretly to accumulate alleged instances of employee wrongdoing, avoiding any notice to the Union [within reasonable delay], and then to aggregate them and reveal the details only when the discharge is challenged is the quintessence of the very unfairness which the parties explicitly sought to prevent by the terms of article XIV...» in Adrian College and Local 547, International Union of Operating Engineers $A F L-C I O, 89$ LA 857, p. 861.

Il est vrai qu'en l'espèce l'arbitre a invoqué une clause conventionnelle; mais il est bien établi que le concept de juste cause inclut, en droit américain, des exigences procédurales telles que l'obligation d'entendre le salarié avant de lui imposer une sanction, de même que le principe de progressivité. Or, la progressivité des sanctions implique l'imposition des mesures disciplinaires au fur et à mesure de la connaissance des manquements du salarié. Contra, en matière de congédiement administratif: Centre Hospitalier Rouyn-Noranda c. Syndicat professionnel des infirmières et infirmiers de Montréal, D.T.E ${ }^{\circ}$ 88T-458, p. 21. 
Pour les motifs d'équité procédurale, les mesures disciplinaires ne peuvent non plus être données «à répétition» ou «en rafale» car alors le principe de progressivité ne peut jouer son rôle: In Re Standard Shade Roller Division and United Paperworkers, Local 860, 73 LA 86; In Re Gill Studio Inc. and Pictorial Painters, Local Union No. 820, 78 LA 916.

38 Re Motor Transport Industrial Relations Bureau of Ontario and General Truck Drivers' Union, Local 938, (1974) 4 L.A.C. (2d) 362.

L'arbitre invoque la théorie de la relation entre l'agent et son principal (la théorie du mandat, en common law). Naturellement, il ne s'agit pas ici d'estoppel by agency, une variété de l'estoppel by representation; en effet, le salarié ignorant l'existence de l'agent ne peut prétendre qu'à travers celui-ci l'employeur lui ait fait des représentations. L'arbitre a plutôt jugé que l'employeur, en engageant un enquêteur, devait lui donner des instructions conformes à ses propres obligations conventionnelles (p. 368).

Le même raisonnement vaut évidemment pour les obligations légales ou extra-contractuelles de l'employeur; la règle tient même en l'absence de clause conventionnelle, à condition d'admettre préalablement la thèse du délai raisonnable.

39 Art. 1701 C.c. et seq.

40 La théorie de la délégation de pouvoir ressortit au droit public et, à cet égard, n'est pas directement applicable à l'entreprise privée. Toutefois, elle correspond mieux que le mandat contrat convenu entre deux personnes - à la notion d'entreprise, entité complexe dont l'autorité est partagée entre une multitude de personnes, par opposition à celle qu'administre un propriétaire-employeur.

Sur la délégation de pouvoir, consulter: Patrice GARANT, Droit administratif, deuxième édition, Montréal, Les Éditions Yvon Blais, 1985, pp. 263 et seq.

41 Voir l'article 1023 C.c.

42 Voir par exemple: Re Falconbridge Nickel Mines Ltd. and Sudbury Mine, Mill and Smelter Workers' Union, Local 598, (1982) 4 L.A.C. (3d) 274; Re Atomic Energy of Canada Ltd. (Chalk River Nuclear Laboratories) and Office \& Professional Employees Union, Local 404, (1982) 5 L.A.C. (3d) 248; Re General Tire Canada Ltd. and United Rubber Workers, Local 536, (1983) 7 L.A.C. (3d) 238.

La jurisprudence québécoise exige elle aussi que le salarié fautif soit prévenu des conséquences fâcheuses qu'entraînerait la non-correction du manquement: Association des employés de Gailuron de Valleyfield Inc. c. Monette et Larivière Inc., [1983] T.A. 236, p. 239; Ville de Maniwaki c. Syndicat des employés municipaux de la Ville de Maniwaki (CSN), D.T.E. $\mathrm{n}^{0} 85 \mathrm{~T}-963$, p. 22; Crane Canada Inc. - Poterie du Québec c. L'Union internationale des travailleurs $d u$ verre, poterie, plastique et autres, local no. 78, 7 C.L.A.S. 131, No. 07:0735, pp. 30-31.

Si le salarié connaît son insuffisance et ses conséquences, ce fait est un facteur aggravant du dossier antérieur: Velan Inc. c. Syndicat national des employés de Velan CSN, DTE $n^{\circ}$ 86T-11. Par ailleurs, dans le cadre du contrat individuel de travail à durée indéterminée, si le salarié est renvoyé brusquement alors que selon toute apparence, sa compétence et son rendement étaient jusque-là satisfaisants, le tribunal pourra conclure à l'absence de juste cause: Miron Inc. c. Des Cheneaux, D.T.E. $\mathrm{n}^{\circ}$ 88T-14, Cour d'appel, district de Montréal, dossier $\mathrm{n}^{\circ}$ 500-09-015304-817, le 11 novembre 1987, pp. 3-4. Faire savoir au salarié les griefs que l'employeur lui reproche apparaît comme une condition nécessaire mais non suffisante de la juste cause.

Selon nous, la condition essentielle est que le salarié sache que sa conduite est fautive aux yeaux de l'employeur et connaisse les conséquences de la persistance de cette faute. Cette information peut découler d'un règlement intérieur clair ou de la convention collective. La nature fautive de l'acte peut même être présumée connue: c'est le cas d'actions que réprouve la loi naturelle i.e. la conscience de l'homme moyen. 
43 Voir, par exemple, la décision Falconbridge, citée à la note précédente, à la page 278. Ne pas prévenir le salarié serait inéquitable («unfair»).

Il ne s'agit pas ici du devoir d'équité procédurale (duty to act fairly) qui, en droit administratif, oblige l'autorité publique à informer un administré (qui en certains cas est un employé de l'État que l'on veut démettre de ses fonctions) des motifs d'une décision, tout en lui donnant l'occasion de faire valoir son point de vue.

44 Voir la décision General Tire, précitée, note 42, p. 244.

Sur l'obligation de prouver l'irréversibilité de l'insuffisance professionnelle, voir: Coca-Cola Limitée c. Union des routiers, travailleurs des brasseries, liqueurs douces et ouvriers de diverses industries, (1981) S.A.G. 263, p. 271; F.F. Soucy Inc. c. Le Syndicat canadien des travailleurs du papier, Locaux 625 et 90, (1981) S.A.G. 936, p. 942; Re Victoria Hospital, London and London and District Building Service Workers' Union, Local 220, (1981) 24 L.A.C. (2d) 172, p. 175; Syndicat des employés de Celanese (CSN) c. Celanese Canada Inc., [1985] T.A. 281 , p. 293.

Noter cependant qu'un courant jurisprudential veut que l'employeur soit plutôt tenu de prouver qu'il n'a pu trouver un travail adapté aux capacités diminuées du salarié. Voir, par exemple: Dominion Textile Ltée c. Union des ouvriers du textile et du coton, (1981) S.A.G. 107.

45 Nous avons relevé, dans les années récentes, une décision qui retient que l'employeur n'a qu'à établir l'insuffisance passée; par présomption de fait, on peut alors inférer que cette situation se reproduira dans le futur. Il y a alors renversement du fardeau de la preuve à la charge du salarié: Re Niagara Structural Steel (St-Catharines) Ltd. and United Steelworkers, Local 7012), (1978) 18 L.A.C. (2d) 385, à la p. 392. Cette règle fut posée dans le contexte d'un manquement continu, en l'espèce l'absentéisme chronique. La discussion porte donc davantage sur l'administration des preuves et le renversement qui s'opère une fois qu'une partie a présenté sa preuve que sur le fardeau de la preuve lui-même.

Au Québec, voir par exemple Le Syndicat des imprimeurs de St-Jean (CSN) c. Éditions du Richelieu Ltée, (1974) S.A.G. 394 dans lequel l'arbitre impose le fardeau de la preuve de l'incapacité à l'employeur. Il semble par ailleurs acquis que la permanence de l'incapacité doit toujours être établie: Hilton International Québec c. Syndicat des travailleurs et travailleuses du Hilton Québec, D.T.E. Nº 86T-531, pp. 18-19.

46 Sur ce développement récent de la Common law, voir: David HARRIS, Wrongful Dismissal, Don Mills, Ontario, Richard De Boo Publishers, 1987, pp. 3-119 et seq et Supplement, December 1987, pp. 60 et seq.

Sur la nécessité de démontrer que le salarié congédié pour rendement insatisfaisant connaissait la norme, voir: Ville de Maniwaki $\mathrm{c}$. Syndicat des employés municipaux de la Ville de Maniwaki, D.T.E. $n^{\circ} 85 T-963$, pp. 13 et 15 à 17; Service central (Québec) Inc. c. Travailleurs canadiens de l'automobile, Section locale 1044, D.T.E. $n^{\circ} 88 \mathrm{~T}-151$, notamment à la page 16 , premier alinéa.

47 Re United Steelworkers and Steel Co. of Canada Ltd., (1972) L.A.C. 221; Re Canadian Appliance Manufacturing Co. Ltd. and United Steelworkers on America, Local 3129, (1979) 19 L.A.C. (2d) 354 (où l'arbitre approuve la décision Steel Co., pp. 357-8); Syndicat national des travailleurs des Produits calcaires Bedford Inc. c. Produits calcaires Bedford Inc., décision non rapportée, le 16 juin 1986, Pierre Cloutier, p. 22 (mesure non disciplinaire approuvée en principe, mais annulée dans les circonstances).

48 Re International Association of Machinists, Lodge 1703 and Perfect Circle - Victor Division, VNG Parts Ltd., (1972) 24 L.A.C. 380; Re Great Atlantic and Pacific Ltd. and Retail, Wholesale and Department Store Union, Local 414, (1982) 3 L.A.C. (3d) 403. Dans ce dernier cas, l'arbitre cite, à la page 411 , cinq décisions américaines relatives à des cas de même espèce (rendement insuffisant par rapport au standard établi). 
Toutefois, la jurisprudence américaine est nuancée sur ce point. Voir, par exemple: Adrian College, 89 LA 857. Dans cette affaire, l'arbitre a décidé qu'un employeur ne pouvait, au bout de quatre mois durant lesquels on avait observé et noté ses manquements, renvoyer un salarié sous prétexte de rendement insatisfaisant en arguant qu'il ne s'agissait pas d'un congédiement disciplinaire. En l'espèce, l'insouciance était la cause du problème. L'arbitre a distingué cette cause d'une incapacité physique ou mentale de faire le travail, auxquels cas l'employeur n'aurait pas été astreint à la règle de la discipline progressive.

Au Québec, il y a aussi un courant qui laisse à l'employeur le choix de procéder suivant la ligne disciplinaire ou non disciplinaire. Par exemple: Sous-vêtements Union, Canada Inc. c. Syndicat des travailleurs de l'énergie et de la chimie, Local 119 (FTQ), décision non publiée, le 11 janvier 1988, J.-P. Lussier, arbitre, pp. 12-14.

49 Re Union Gas Ltd. and Oil, Chemical \& Atomic Workers' International Union, (1979) 21 L.A.C. (2d) 437.

so Cette étude n'est à proprement parler ni doctrinale, ni jurisprudentielle, au sens où elle ne prétend pas être exhaustive ni même représentative; conséquemment, elle ne fait pas le point sur l'état actuel du droit et, sur la question précise qui nous occupe, on ne peut donc parler ni d'école doctrinale, ni de courant jurisprudentiel.

Tout au plus, peut-on parler, de diversité de la règle de droit qui paraît n'être pas fixée. Les remarques qui suivent doivent s'interpréter dans ce contexte d'incertitude.

51 En droit commun, l'employeur peut rompre brusquement le lien d'emploi s'il y a un motif réel et sérieux, tout en demeurant seul juge de la proportionnalité entre le manquement et la sanction (abstraction faite de tendances jurisprudentielles récentes).

52 La mutation et la rétrogradation ne sont pas universellement admises en tant que mesures disciplinaires. Elles sont incontestablement permises en matière non disciplinaire. D'autres mesures, telle la suspension sans solde, sont admises en tant que sanction d'un manquement disciplinaire ou non disciplinaire.

53 Re Teamsters Union, Local 880 and Reimer Express Line Ltd., (1957) 7 L.A.C. 341, pp. 344-5. L'arbitre reprend à son compte la relation de l'état de la common law que l'on trouve dans: Canadian Encyclopedic Digest (Ontario), Second edition, Volume 13, Toronto, The Carswell Company Ltd., 1955, pp. 23-4. (Le Supplement de 1987 donne, sur la question, le même compte rendu de la common law, p. 70, No. 112.) Voir aussi: Re Sudbury Mine, Mill and Smelter Workers Union, Local 598 and Falconbridge Nickel Mines Ltd., (1958) 8 L.A.C. 243, p. 245 (et la jurisprudence citée); Re Air Canada and Canadian Air Line Employees' Association, (1982) 4 L.A.C. (3d) 69, p. 75.

54 Howard A. LEVITT, The Law of Dismissal in Canada, Aurora, Ontario, Canada Law Book Inc., 1985, pp. 127-28.

55 Voir: Innis CHRISTIE, Employment Law in Canada, Toronto, Butterworths, 1980, pp. 369-70; Richard HARRIS, Wrongful dismissal, Don Mills, Ontario, Richard De Boo Publishers, 1984, pp. 3-132 et seq.; Ellen E. MOLE, Wrongful Dismissal Practice Manual, Volume I, Scarborough, Ontario, Butterworths, 1984, pp. 423-24.

56 Re Corporation of the District of Burnaby and C.U.P.E., Local 23, (1984) 11 L.A.C. (3rd) 418, pp. 422-3. En doctrine, voir: Donald J.M. BROWN and David M. BEATTY, Canadian Labour Arbitration, Second edition, Aurora, Ontario, Canada Law Book, 1984, pp. 477 et seq.

Au Québec, voir: La Cité de St-Léonard c. Association des pompiers de St-Léonard, (1981) S.A.G. 2121, p. 2127; Liquid Carbonic Inc. c. Syndicat des Métallurgistes Unis d'Amérique, décision non rapportée, le 26 juin 1987, Léopold Lavoie, p. 11.

57 Re Zehrs Markets, A Division of Zehrmart Ltd. and Retail Clerks' Union, Local 1977, (1986) 23 L.A.C. 124. Cette décision est aussi à l'effet que, sur le plan des règles d'administration de la preuve, l'employeur n'est pas admis, en contre-preuve, à établir les manquements passés (et impunis) du salarié, si le salarié a nié, en contre-interrogatoire, ses erreurs 
passées car cette dénégation ne résulte pas de son initiative. Observons qu'autrement autant jeter la règle par-dessus bord puisque l'employeur pourrait, à son gré, la contourner. Voir aussi l'arrêt Corporation of the District of Burnaby, note précédente, pp. 427-8.

58 Voir: Re Commercial Telegraphers' Union and Canadian Pacific Railway Co., (1971) 22 L.A.C. 312; Re City of Vancouver and Vancouver Municipal and Regional Employees Union, (1984) 11 L.A.C. (3d) 122; Re McKellar General Hospital and Canadian Union of Public Employees, Local 1409, (1986) 24 L.A.C. (3d) 69.

Les mêmes remarques valent pour la rétrogradation fondée sur l'insuffisance professionnelle: Re Labatt Ontario Breweries, Division of Labatt Brewing Co. Ltd. and Canadian Brewery Workers Union, Local 304, (1981) 29 L.A.C. (2d) 275.

59 Voir: Re Edith Cavell Private Hospital and Hospital Employees' Union, Local 180, (1982) 6 L.A.C. (3d) 229, p. 233; Re McKellar General Hospital and CUPE, Local 1409, note précédente, pp. 74-75.

Dans l'affaire McKellar General Hospital, l'employeur avait cherché en vain un poste approprié aux aptitudes du salarié avant de le congédier. Il l'avait, par la suite, invité à poser sa candidature à un poste devenu disponible, mais le salarié n'avait pas réagi, «foolishly» selon l'arbitre. Il décide néanmoins que l'employeur eût dû faire un effort exceptionnel («extraordinary steps") pour lui trouver un poste convenable.

60 Notre jurisprudence est assez permissive quant à la discrétion qu'a l'employeur d'attribuer telle fonction à tel individu ou d'inclure telle tâche dans telle fonction. La limite supérieure de cette marge de manoeuvre correspond à la notion de congédiement déguisé: Robert $P$. GAGNON, Louis LeBEL et Pierre VERGE, Droit du travail, Québec, P.U.L., 1987, p. 141, et la jurisprudence citée aux notes 36 et 37. Voir cependant: Cherry Reilly c. Hotel of Distinction Canada (Inc.) Hotel Le Grand/Grand Hotel, [1987] R.J.Q. 1003, en appel, dossier $\mathrm{n}^{\circ}$ 500-09-000752-873 (il y a congédiement déguisé quand l'employeur confie à un nouvel employé certaines fonctions de direction jusque-là dévolues à une salariée et lui enlève certains privilèges).

De même, en France, la jurisprudence s'est montrée très réceptive à la révision du contrat de travail. Voir (1986) Droit social, $\mathrm{n}^{\circ} 12$, numéro spécial sur la précarité des avantages acquis.

61 Naturellement, les clauses d'ancienneté devraiein être adaptées en conséquence.

62 L'exécution normale ou diligente est celle du travailleur moyen, placé dans les mêmes circonstances. Ainsi, le salarié débutant n'est pas tenu au rendement du travailleur pleinement qualifié, le travailleur en fin de carrière n'est pas tenu au rendement qu'il devait fournir lorsqu'il est parvenu à maturité, etc.

63 Cela ne signifie pas qu'à l'enquête, la preuve des fautes antérieures soit irrecevable. En effet, la suffisance de la cause de renvoi ne sera déterminée qu'après coup, lors du prononcé du jugement, trop tard pour apporter une preuve de manquements antérieurs qui aurait été omise.

64 La notion d'amnistie (ou condonation), qui retient la faveur des arbitres, appartient au droit disciplinaire, tandis que la théorie de la révision ressortit au droit des contrats.

65 Sur l'amnistie des fautes sanctionnées voir: Bruce YOUNG, Beyond Discharge, Toronto, The Canada Labour Views Co. Limited, 1984, pp. 253 et seq. L'auteur étudie les clauses d'amnistie en regard du principe de progressivité et de la doctrine de l'incident culminant.

66 Nous ne traiterons pas ici de l'appréciation d'une faute plus ou moins lointaine dans un dossier par ailleurs irréprochable. C'est là une circonstance dont l'arbitre peut tenir compte (art. 100.12 f) C.t.). À ce titre, la question est un cas particulier du problème plus général du processus décisionnel dans le cadre de l'arbitrage ou, si l'on veut, d'une théorie de l'appréciation des circonstances par les arbitres. On trouvera une illustration intéressante de ce point 
dans: Re Government of Alberta v. AUPE Harding, 8 C.L.A.S. 9, No. 08:0056. Dans cette affaire, le tribunal décide que le salarié ne bénéficie pas de la clause d'amnistie, mais que la faute étant très ancienne, la preuve bien qu'admissible n'a que peu de poids.

Sur le principe de l'importance décroissante de la faute avec le temps: Re United Automobile Workers, Local 112 and Douglas Aircraft of Canada Ltd., (1968) 18 L.A.C. 27; Re Steel Co. of Canada Ltd. and United Steelworkers, Local 1005, (1975) 8 L.A.C. (2nd) 213, p. 218; Re Canadian Lukens Ltd. and United Steelworkers of America, (1976) 12 L.A.C. (2d) 439 , p. 447.

67 Au Québec, au 31 décembre 1986, environ 79\% des conventions collectives en vigueur comportaient une clause d'amnistie et ce chiffre était de $93 \%$ pour les conventions signées entre le premier septembre 1985 et le 31 août 1986. Source: Conditions de travail contenues dans les conventions collectives, Québec, 1986, Centre de recherche et de statistiques sur le marché du travail, ministère du Travail, Gouvernement du Québec, 1987, pp. 70-71.

On trouve aussi des clauses d'amnistie aux États-Unis. Voir: Schreiber Trucking Co. Inc. and Teamsters Union Local 6610, 48 LA 833; United States Steel Corporation, Sheet and Operations, Pittsburg Works and United Steel Worker of America, Local 1440, 53 LA 126; In re City of Quincy, Illinois and International Association of Machinists, Local Lodge 822, 81 LA 352 , p. 359.

68 Parmi les conventions comprenant une clause d'amnistie, 46,2\% (1 376 sur 2977 ) prévoyaient l'expurgation du dossier après douze mois ou moins, sans condition.

D'autres ajoutent, au simple écoulement du temps, une autre condition pour l'acquisition de l'amnistie; cette condition est invariablement l'absence de faute durant la période retenue.

Certaines conventions font varier la période de temps requise en fonction de la nature du manquement. Source: ibid., pp. 70-71.

69 Cette assertion n'est pas à l'abri de tout débat. Ainsi, Le petit Larousse illustré, Paris, Librairie Larousse, 1986, indique clairement que "récidiver» consiste à "commettre de nouveau la même faute, le même délit, le même crime». Mais la définition de «récidive» permet d'y inclure la commission d'une nouvelle infraction, quelle qu'en soit la nature, après une première condamnation. Les définitions correspondantes du Petit Robert, Paris, Société du Nouveau Littré, 1981, prêtent flanc à la même discussion. Dans ces conditions, irait-on jusqu'à dire qu'une clause aurait une portée différente suivant qu'elle se lirait «à condition de ne pas récidiver» plutôt qu' «à condition qu'il n'y ait pas de récidive»? Ce serait sans doute exagéré. Par contre, il y aurait certes lieu de distinguer une condition de non-récidive de celle, plus générale, de ne pas commettre d'autres fautes ou manquements subséquemment, quelle qu'en soit la nature.

Signalons, enfin que le droit pénal français distingue les deux variétés de récidive. Cf. Gérard CORNU (sous la direction de), Dictionnaire juridique, Association Henri-Capitant, Paris, P.U.F., 1987.

Sur la notion de récidive, voir: La Presse Limitée c. Syndicat des travailleurs de l'information de La Presse Ltée (CSN), [1983] T.A. 291, p. 298; Ville de Sainte-Thérèse c. Fraternité des policiers de Ste-Thérèse Inc., D.T.E. $\mathrm{n}^{\circ} 88 \mathrm{~T}-337, \mathrm{p} .12$ (1'arbitre décide que la récidive comprend tout manquement ultérieur).

70 Sur l'amnistie en fonction de la distinction entre mesures disciplinaire et non disciplinaire, voir: Hilton International-Québec c. Syndicat des travailleurs et travailleuses du Hilton Québec, D.T.E. $\mathrm{n}^{\circ}$ 87T-531 (les avis d'inconduite, prescrits au plan disciplinaire, sont admissibles au plan «administratif» i.e. pour établir l'incapacité professionnelle du salarié); Société Radio-Canada c. SCFP (Conseil des sections locales de la DSF, (Groupe des employés de bureau et professionnel), [1986] T.A. 660, p. 673 (le dossier d'absentéisme et des certificats médicaux s'y rapportant ne constituent pas en eux-mêmes des mesures disciplinaires; ils ne sont donc pas couverts par une clause d'amnistie disciplinaire et sont admissibles en preuve). 
71 Du reste, nous avons vu, plus haut, que l'auteur d'une faute non disciplinaire doit être prévenu que son manquement doit être corrigé et, qu'à défaut de le faire, le salarié mettrait son poste en péril. Cette «discipline progressive» ne comporte que deux paliers, contrairement à la progressivité proprement dite qui peut comporter plusieurs degrés de sanction.

Il semblerait logique, dans le système à deux paliers, que l'employeur doive recommencer à zéro si un manquement de nature différente se produit; autrement, comment le salarié saurait-il que sa prestation est impropre et qu'elle met son poste en péril? Ceci dit, il n'est pas facile, en pratique, de distinguer entre un manquement dû à des causes successives différentes (e.g. absentéisme chronique dû à la maladie, puis à un accident, ensuite à l'incendie de sa maison, etc.) et des manquements différents dûs à une même cause (e.g. l'alcoolique qui est hospitalisé épisodiquement, qui cherche souvent querelle à ses compagnons de travail, qui a un taux d'accidents plus élevé que la moyenne, etc.).

Cette progressivité à deux paliers - en cas de manquements non disciplinaires, parfois appelés fautes contractuelles - correspond à l'autorité disciplinaire à deux paliers, avertissement et renvoi, en droit commun. En droit disciplinaire proprement dit, la progressivité des sanctions est graduelle et repose sur le postulat implicite que, s'agissant de «distinguer entre le bien et le mal», le salarié sait d'instinct ou par nature quels agissements sont fautifs et que, d'autre part, tous les comportements fautifs relèvent d'une même catégorie i.e. ce qui est prohibé. C'est pourquoi dans l'application du principe de progressivité, l'identité de nature des fautes disciplinaires successives n'est pas une condition nécessaire bien qu'elle constitue à l'occasion un facteur à considérer dans l'appréciation de la juste cause.

Cela n'empêcherait pas un employeur de juger, éventuellement avec l'approbation de l'arbitre, que : elle faute est d'autant plus grave qu'elle est de même nature que celle qui apparaissait déjà à son dossier, notamment en ce qui concerne la rupture du lien de confiance.

Sur le pouvoir disciplinaire de l'employeur en common law, voir: Donald J.M. BROWN and David BEATTY, Canadian Labour Arbitration, Second Edition, Aurora, Ontario, Canada Law Book Limited, 1984, pp. 330-331. Sous ce régime, l'employeur n'a que le pouvoir de congédier pour cause; l'amende, la suspension sans salaire, etc. ne lui sont pas ouverts. Par ailleurs, il est évident que, n'étant pas obligé de congédier, même si une cause suffisante existe, il lui est loisible de recourir à l'avertissement, une mesure moins sévère.

72 Voir: Pierre MARTINEAU, La prescription. Traité élémentaire de droit civil, Montréal, P.U.M., 1977, nº 177.

Nous raisonnons comme si, après l'écoulement de la période prévue, le salarié acquérait le droit d'exiger que son dossier soit expurgé. Mais un autre raisonnement peut être tenu: après le délai, le droit de l'employeur d'opposer sa faute au salarié s'éteint. Il s'agit alors de prescription extinctive. Dans un cas comme dans l'autre, la computation des délais suit les mêmes règles: ibid., $\mathrm{n}^{\circ} 246$.

Une analyse axée sur le droit contractuel satisfait davantage l'esprit, sans pour autant changer la solution. En vertu de la clause d'amnistie, l'obligation de l'employeur d'expurger le dossier nait dès la commission de la faute (ou de l'imposition de la sanction, suivant ce qui est convenu), mais l'exécution n'en peut être exigée avant l'arrivée du terme suspensif.

Le calcul du délai demeure inchangé, quelle que soit l'analyse retenue. Par conséquent, il n'y a pas à s'inquiéter du point de départ du délai. Celui-ci court automatiquement, sauf disposition contraire dont on trouvera un exemple dans: The Government of the Province of Alberta v. The Alberta Union of Provincial Employees, 8 C.L.A.S. 9, No. 08:0056 (la convention collective édictait clairement que l'expurgation était faite à la demande du salarié et que l'amnistie prenait effet à compter de cette expurgation, pp. 2 et 3 ).

73 La preuve d'une faute antérieure est une condition nécessaire, quoique non suffisante, à l'argumentation fondée sur la doctrine de l'incident culminant. Ainsi en est-il lorsqu'à la succession de fautes l'employeur attache la conséquence de la destruction du lien de confiance. 
74 Henri LEON et Jean MAZEAUD, Leçons de droit civil, tome deuxième, sixième édition par Jean CHABAS, Paris, Édition Montchrestien, 1978, $\mathrm{n}^{\circ} 1024$.

75 Le problème de la maladie s'est posé dans: Ville de Maniwaki c. Syndicat des employés de la Ville de Maniwaki (CSN), D.T.E. $\mathrm{n}^{\circ}$ 85T-963. Dans cette affaire, il est vrai que le délai avait couru sans égard à l'absence prolongée du salarié; un délai d'amnistie plus long, ou une règle en suspendant le cours, auraient pu produire un résultat différent. Le supérieur du salarié a tout de même tranché le fond de la question en soutenant que «'absence de plainte contre le plaignant au cours de l'année 1982 s'expliqu[ait] du fait que ce dernier a été absent du travail, en congé maladie, pendant toute l'année» (p. 7). Cela revenait à dire que s'il avait été présent, il aurait commis d'autres fautes (mais il y a là une imputation d'intentions déplaisante et, au mieux, une hypothèse non vérifiable) ou encore qu'une absence prolongée ne devrait pas entrer en compte dans le calcul du délai (encore que dans ce cas d'espèce, l'amnistie aurait été quand même acquise).

Par ailleurs, il a déjà été décidé, en présence d'une clause ambiguë et d'une pratique établie en ce sens, qu'une période d'absence en raison de maladie devait être exclue du calcul: Re International Union, United Automobile, Aerospace \& Agricultural Implement Workers, Local 1967 and McDonnell Douglas Canada Ltd., (1984) 47 O.R. (2nd) 78 (High Court of Justice, Divisional Court).

76 Ces événements ne relèvent pas les parties de toutes leurs obligations. Par exemple, le salarié demeure tenu à son obligation de loyauté. Voir cependant l'arrêt La Compagnie de sable Ltée, c. Commission des normes du travail, [1985] C.A. 281, p. 285 où il est affirmé qu'en cas de mise à pied, «[l]a nature des relations juridiques qui subsistent est tellement modifiée que le lien contractuel n'impose plus aux parties les mêmes obligations»".

77 Robertson Building Systems Limited, and International Association of Bridge, Structural and Ornemental Iron Workers, Local 734, (1985) 19 L.A.C. (2d) 427. Dans cet arrêt, l'arbitre a décidé que les délais se mesuraient en unités de temps civiles, à défaut de précisions, et qu'une mise à pied temporaire ne suspendait pas l'écoulement du temps.

78 Voir P. MARTINEAU, op. cit., p. 209.

79 Le recours aux règles de la prescription — règles légales — n'est pas sans limites, car il s'agit ici d'un délai conventionnel dont les parties peuvent fixer les règles à leur gré.

80 Dans l'article 1019, «stipuler» a son sens originaire signifiant «acquérir des droits par convention»: Gérard CORNU (sous la direction de), Vocabulaire juridique, Association Henri-Capitant, Paris, P.U.F., 1987.

81 Comparer: Jean-Louis BAUDOUIN, Les obligations, Cowansville, Les Éditions Yvon Blais, 1983, p. 231.

Notons que cette approche ne fait pas l'unanimité chez les arbitres. Relativement à une clause conditionnant l'opposabilité de manquements passés à l'observation de certaines formalités au moment où ils se sont produits - et dans cette mesure la clause examinée se comparait à une clause d'amnistie - un arbitre, dans une décision élaborée, a opté pour la solution la plus favorable au salarié: Re City of Vancouver and Vancouver Municipal and Regional Employees Union, (1984) 11 L.A.C. (3d) 121, p. 134.

82 Hôpital St-Charles-Borromée et Syndicat des travailleurs(euses) du Centre Hospitalier St-Charles-Borromée, A.H.Q., ${ }^{\circ}$ 328-03-07, le 23 avril 1982, p. 6.

83 Sur le plan analytique, la question de cette renonciation n'est pas si simple. S'agit-il de remise d'une obligation? le consentement de l'employeur serait alors nécessaire: Jean-Louis BAUDOUIN, $o p$. cit., p. 490. Or, s'il est avantageux pour le salarié de «renoncer», sans doute l'est-il tout autant pour l'employeur de laisser la convention suivre son cours normal, done de refuser son consentement.

Par ailleurs, il est difficile de voir ici une renonciation proprement dite, i.e. un acte juridique unilatéral. Sur la complexité de la notion de renonciation, voir: Pierre RAYNAUD, «La 
renonciation à un droit», (1936) Revue trimestrielle de droit civil 763; Yves SEILLAN, «L'acte abdicatif», (1966) Revue trimestrielle de droit civil 686; Paul ROUBIER, «Les prérogatives juridiques», (1960) Archives de philosophie du droit 65.

Enfin, cette «renonciation» au bénéfice de la clause ayant pour objet un événement particulier, elle n'a pas d'effet pour le futur; aussi, la notion de faculté ne semble pas utile à l'analyse de cette question: Jacques FLOUR et Jean-Luc AUBERT, Les obligations. L'acte juridique, Paris, Armand Colin, 1975, $\mathrm{n}^{\circ} 375$.

84 Si un salarié n'a commis qu'une seule faute, qu'elle remonte à plusieurs années audelà de la période d'amnistie et que, par ailleurs, il compte de longs états de service, alors la clause d'amnistie ne lui est pas d'un grand secours. En effet, une faute passée s'estcompe naturellement avec le temps et elle disparaît d'autant plus rapidement qu'elle est moins grave.

Pour un exemple intéressant, voir: Re Steel Co. of Canada, Hilton Works and United Steelworkers of America, Local 1005, (1980) 26 L.A.C. (2d) 411. (Noter, incidemment, que cet arrêt reconnaît l'usage de l'entreprise comme source du droit à l'amnistie).

Voir également: The Government of Alberta v. The Alberta Union of Provincial Employees (Harding), 8 C.L.A.S. 9, No 08:0056 (le tribunal décide que la preuve d'une faute étant admise parce que le salarié n'avait pas demandé l'expurgation de son dossier, cette preuve n'aura pas une grande force probante, vu que la faute remonte à plusieurs années, pp. 4-5).

85 À l'arbitrage, la question se présentera vraisemblablement sous l'angle du droit de la preuve. Ainsi, l'acceptation par l'employeur de la renonciation se fera tacitement, le syndicat mettant en preuve le dossier du salarié sans opposition.

Cela étant, le syndicat ne pourra par la suite s'opposer à une preuve tendant à contredire la sienne, en l'occurrence à établir les faits tendant à démontrer que le dossier n'est pas aussi favorable que le réclamant le prétend. Cela découle de la règle audi alteram partem à laquelle l'arbitre est tenu strictement. En pratique, le principe trouvera application par le moyen du contre-interrogatoire.

86 Voir: Re Wire Rope Industries Ltd. and United Steelworkers of America, (1985) 17 L.A.C. (3d) 409; Re B.C. Rail Ltd. and United Transportation Union, Locals 1178 and 1923, (1985) 17 L.A.C. (3d) 402; Re District of Burnaby and Canadian Union of Public Employees, Local 23, (1983) 11 L.A.C. (3d) 418; Re City of Vancouver and Vancouver Regional Employees Union, (1983) 11 L.A.C. (3d) 121; Ingersoll-Rand Canada Inc. c. Métallurgistes Unis d'Amérique, Local 6670, S.A. 83-12-234, le 13 décembre 1983, M. Boisvert.

87 Industrial Relations Act, R.S.B.C. 1979, c. 212.

88 Re Wire Rope Industries Ltd., précité, note 86 à la p. 413:

[...] Section 98(d) requires an arbitrator to exercise his remedial authority «to determine [whether] a dismissal or discipline is excessive in all the circumstances of the casem. Nowhere does the Labour Code specify what are the relevant circumstances of each case. Nor does the Code direct the parties as to what offences might be considered grounds for discipline. As we read art. 10.12, it prevents an arbitrator from considering certain items of an employee's disciplinary record for purposes of determining whether the employer has just cause to discipline or discharge an employee. In effect, the parties have indicated that an employee's discipline record that is more than 12 months old is simply not relevant, i.e., is not to be a circumstance which the arbitrator should consider in exercising his remedial authority under $s$. 98(d). In our view, the remedial authority of the arbitrator has not been usurped by the parties by the insertion of art. 10.12 into their collective agreement. An arbitrator has full authority to consider whether a particular disciplinary response is excessive and if so what should be the appropriate penalty. This authority to substitute a penalty has not been circumscribed in any way. What art. 10.12 does is simply to indicate what evidence an arbitrator may refer to in exercising his remedial authority. In so many words, the parties have indicated (to use the language of s. 98(d)) that a discipline record that dates back in excess of 12 months is "not a circumstance of the caser. 
89 L.R.Q. c. C-27.

90 Arrêt Ingersoll-Rand, précité, note 86, p. 9.

Contra: Bombardier Limitée, Division Caoutchouc c. Le Syndicat des salariés de Bombardier Limitée, Division Rockland, SA 80-10-274, Claude Fabien, citée dans la décision Ingersoll-Rand, pp. 10-11. On y distingue la preuve de la sanction de celle de la faute, cette dernière étant admissible en dépit d'une clause d'amnistie. Mais n'est-ce pas là vider cette clause de toute finalité? D'autre part, à la limite, l'impossibilité de prouver la sanction de la faute passée empêche de prouver la progressivité des sanctions et donc intervient dans l'appréciation de la proportionnalité entre la faute la plus récente (celle qui fait l'objet du grief) et sa sanction. Mais cela revient à dire qu'il ne peut être tenu compte de la faute antérieure et c'est justement le but de la clause d'amnistie.

91 United States Steel Corp., 53 LA 124.

En l'espèce, la clause visait spécifiquement la preuve des fautes remontant à plus de cinq ans; l'arbitre conclut qu'il n'était pas nécessaire d'examiner le fond (merit) du grief, même si l'employeur ne s'était pas appuyé sur les fautes passées pour décider de la mesure disciplinaire appropriée.

\section{Labor Studies Journal}

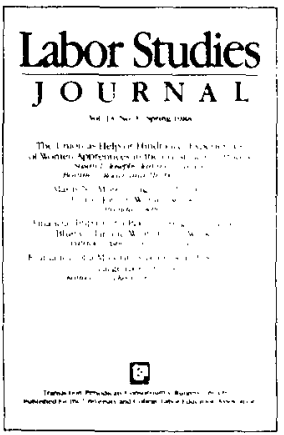

Richard Humphreys, edhor

(West Virginia University)

Explores the role of the trade union movement in forging American economic and social policy. Provides basic intormation on the philosophy and substance of labor education.

\section{Recent articles Include:}

Thomse DuBole and Bruce Niseen on changing industrai relations in steel.

Randy Hodson, Doborah Zlegler and Barbara

Bump analyze the CWA strike of 1983 .

Brian Towers presents a British perspective on

labor education in depression.

Brian Heshizer discusses how union officials

assess the labor movement and labor-

management rolations.

Janet Stern Solomon studies union responses to

tochnological change.

Published Querterly

Officlal fournal of the University and College

Labor Eoucstion Associstion.

Subseription rates:

Individuals: $\$ 30 / y r ; \$ 55 / 2 y r s ; \$ 80 / 3 y r s$

Institutions: $\$ 45 / y r ; \$ 85 / 2 y r s ; \$ 120 / 3 y r s$

Domestic first-cless msil add $\$ 12 y r$.

Fordon eurtece mail add $\$ 12 / y r$.

Forwion alrmall add 525/yr. 